\title{
Hubert Rottleuthner Zur Soziologie richterlichen Handelns (II)
}

Die Bedeutung der Soziologie für die Rechtswissenschaft, diskutiert an deren Focus: richterlichem Handeln, ist unser allgemeines Thema. Dabei geht es zunächst (I) um eine Rezeption sozialwissenschaftlicher Ansätze, insbesondere der handlungstheoretischen Modelle. Es soll die Viclzabl theoretischer Richtungen und Modellbildungen vorgeführt werden. (2) Aber nach welchen Kriterien sind die konkurrierenden Theorien auszuwählen? Ihre strenge wissenschaftliche Uberprïfbarkeit und Erklärungskraft wären nicht die einzigen. Es geht nämlich und das zu betonen liegt nahe, wenn Juristen sich anschicken, soziologische Ansätze zu verarbeiten - um eine letztlich wissenschaftspolitische und moralische Entscheidung, weil notwendig (a) Annahmen über die »Natur des Menschen * als handelndes Wesen gemacht werden müssen: ob er als verdinglichter Attitüden-Träger, Systemfunktionär, sprachliches Wesen, als in Rollen Handelnder angesehen wird, durch Vorverständnis, Unbewußtes, Ideologie verblendet erscheint; (b) weil der Bereich möglicher Informationen vorweg festgelegt wird: verhaltenswissenschaftliche Theorien liefern bedingte Prognosen über Reaktionen von Attitüden-Trägern (s. Teil I, KJ 1970, S. 299 ff.); Systemtheorie sozialtechnische Informationen über Systemsteuerungen; Spieltheorie und Interaktionismus können für den strategischen und kommunikativen Umgang mit anderen anleiten; Psychoanalyse und Ideologiekritik beanspruchen über Lebensgeschichte, Bedürfnisse und soziale Interessen aufzuklären. (3) Die Vielzahl soziologischer Ansätze zeigt, daß die Sozialwissenschaft nicht rein empirisch verfährt, sondern auch normative Bezüge aufweist (s. u. S. 75 ff.) Wenn die Differenz von Sein und Sollen kein taugliches Kriterium zur Abgrenzung von Soziologie und Rechtswissenschaft darstellt, dann wäre zu prüfen, inwieweit nicht auch die Rechtswissenschaft als Sozialwissenschaft zu begreifen ist - und nicht erst mit ihr zu *integrieren " wäre -, die vom Modell symbol- und regelgeleiteten Handelns ausgeht - freilich in "praktischer *, d. h. handlungsorientierender Absicht. (4) Schließlich könnten die vorgestellten Handlungsmodelle eine Diskussion in Gang setzen, in der die Handlungskonzepte, derer sich Juristen bedienen - nicht zur Interpretation ihres eigenen Handelns, sondern desjenigen ihrer Subsumtionsobjekte - problematisiert würden. Die Handlungsmodelle des juristischen Objektbereichs (Wille/Erklärung; strafrechtliche Handlungsbegriffe, Wissen/ Wollen, Schuld; "Eingriffe« in grundrechtliche Exklusivsphären etc.) könnten der etablierten soziologischen Forschung zugeführt werden; die strafrechtlichen Motivationskonzepte ließen sich als so simpel wie repressiv kritisieren. Indem sie vom »bösen Willen « des Normverletzenden ausgehen, erschöpft sich die richterliche Korrektur in Appellen an den "guten Willen * des womöglich zehnfach Vorbestraften, angesichts dessen Lebensgeschichte die richterliche Moral barer Zynismus ist; zumindest so naiv wie die Frage, wann sich die Justiz endlich bessern wolle. 
Spieltheorie ist eine Entscheidungstheorie, die von zwei oder mehr Entscheidenden mit verschiedenen Zielsetzungen ausgeht und zu deren Durchsetzung »rationale» Strategien formuliert. Sie geht von einem Motivationskonzept aus, an das Prinzipien rationalen Handelns geknüpt werden. Das vorausgesetzte Handlungsziel ist die Optimierung von Interessen, die Maximierung meßbarer Größen: von Geld, Gütern, Waffen, Stimmen. Die Spieler, aufgeklärt über ihre und der anderen Wünsche, verfolgen gleiche, aber meist gegenläufige Interessen: ihre Motive sind also transparent und austauschbar. Es besteht weniger Ungewißheit über zu wählende Strategien, als vielmehr ein Risiko darüber, welche der möglichen Strategien der andere einschlägt. Instruktive Maximen definieren optimale Wahrscheinlichkeiten für die Erreichung von Zielvorstellungen. Diese Imperative erlauben keine Voraussagen: sie können übernommen und erfüllt werden; sie sind unabhängig von den empirischen Bedingungen der Umwelt und der subjektiven Motivlage - der persönlichen Wertschätzung, der zeitlichen Instabilität von Präferenzen, subjektiver Ungewißheit. Werden faktische Abweichungen vom idealisierten Modell festgestellt, so läßt sich allenfalls sagen, daß das Handeln nach den Kriterien des Modells «irrational« war.

Diese knappen Ausführungen sollen an den Arbeiten spezifiziert werden, in denen richterliches Handeln spieltheoretisch untersucht wird ${ }^{1}$. Glendon Schubert hält eine Anwendung der Spieltheorie aufs Recht deshalb für naheliegend, weil es sich bei gerichtlichen Auseinandersetzungen um einen rationalen, formalisierten Konflikt handele ${ }^{2}$. Das Ziel, das spieltheoretisch analysiert werden kann, ist nicht eine unquantifizierbare "Gerechtigkeit«, sondern richterliche "Machta; nicht in dem Sinn der Durchsetzbarkeit gerichtlicher Entscheidungen, sondern der innergerichtlichen Einflußmaximierung. Wieder einmal ist der Supreme Court der USA mit seinen neun Richtern das Untersuchungsobjekt. Es geht um "power «-Maximierung in einem Neun-Mann-Kollegium. "Power « ist dabei definiert als *a player's being pivotal in the minimal winning coalition «3. Wer also am häufigsten die entscheidende Stimme in einer möglichst kleinen Gewinnkoalition abgibt, der hat - so die formale Definition - die größte Macht. Das theoretisch mögliche und dann faktisch erreichte $\mathrm{Maß}$ an Macht läßt sich mit dem Shapley-Shubik-Power-Index ermitteln ${ }^{4}$.

1 Samuel Krislov, Power and Coalition in a Nine-Man Body ( 1963$)$, in: Glendon Schubert, ed., Judicial Behavior, Chicago: Rand McNally, 1964, S. ${ }_{461-464}$; Glendon Schubert, Quantitative Analysis of Judicial Behavior, New York: The Free Press of Glencoe, 1959, S. 173-267; ders., Policy Without Law: An Extension of the Certiorari Game (1962), in: Judicial Behavior, S. 4 I $5-442$; ders., The Power of Organized Minorities in a Small Group, ebd., S. $465-476$; Richard C. Snyder, Game Theory and the Analysis of Political Behavior, in: S. Sidney UImer, ed., Introductory Readings in Political Behavior, Chicago: Rand McNally, 1961, S. 27 I-276. - Zurüdkgegriffen wird dabei vor allem auf: J. von Neumann und O. Morgenstern, Theory of Games and Economic Behavior (1944) (dt. Ausgabe: Spieltheorie und wirtschaftliches Verhalten, Würzburg: Physica, 1961); R. Duncan Luce und Howard Raiffa, Games and Decisions, New York: Wiley, 1957. Zur allgemeinen Einführung s. auch: Martin Shubik, Hg., Spieltheorie und Sozialwissenschaften, Frankfurt a. M.: S. Fischer, I965.

2 G. Schubert, N. I (1959), S. 176 .

3 Ebd., S. 186.

4 L. S. Shapley und Martin Shubik, A Method for Evaluating the Distribution of Power in a Commitce System, in: American Political Science Review, 48 (1954), S. 787-792 (dt. in: M. Shubik, N. I, S. 148-157). - Eine nähere Darstellung, auf die ich hier nur verweisen kann, findet sich bei Henning Eikenberg, Voraussetzungen und Schwierigkeiten der empirischen Erforschung richterlicher Entscheidungsgrundlagen, in: Rüdiger Lautmann, Werner Maihofer und Helmut Schelsky, Hg., Die Funktion des Rechts in der modernen Gesellschaft (Bd. I des Jahrbuchs für Rechtssoziologie und Rechtstheorie), Bielefeld: Bertelsmann, 1970, S. 361-379 (S. 374 ff.). 
Ahnlich wie wir die hermeneutische Problematik als Eigentümlichkeit des Objektbereichs als Argument gegen die Unzulänglichkeit verhaltenswissenschaftlicher Ansätze verwendet hatten, können wir auch spieltheoretische Versuche deshalb kritisieren, weil sie Spezifika richterlichen Handelns nicht treffen.

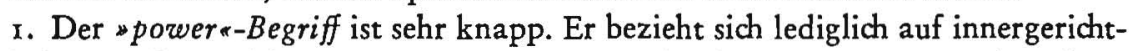
liche Handlungsabläufe, er setzt schon immer Blockbildungen (und Blockanalyse) voraus, er erfaßt nur - und lediglich auf uneinheitliche Entscheidungen gestützt - formale Koalitionsstrukturen. "Macht« wird damit als eine Kategorie der Beziehung von Justiz zur Umwelt eliminiert: dann wäre nämlich zu überlegen, ob nicht gerichtliche Macht durch einheitliche Voten gesteigert wird; maximale innergerichtliche Koalitionen zu bilden, legt aber der verwendete Index nicht nahe. Weiterhin bleiben Bedingungen der Blockbildung ausgespart; dazu wären empirische Untersuchungen über Konsens und Einfluß in Kleingruppen erforderlich. Schließlich bedeutet Macht hier nicht die Durchsetzung von Werten, Anschauungen über public-policy gegenüber unteren Gerichten und der weiteren Umwelt. Macht wird zum unpolitischen Konzept, angewendet auf eine Instanz, die Schubert selbst als politische *power group * begreift.

2. Das Absehen gerade von substantiellen Erwägungen, die Richter nach außen hin in Urteilsbegründungen anstellen, legt die Frage nahe, ob das »power *Konzept überhaupt eine adäquate Motivationskategorie für richterliches Handeln bilden kann. Schubert beansprucht mit seinen erwähnten Arbeiten, politisches Verhalten zu interpretieren, Koalitionsstrukturen und politische Erfolge zu beschreiben; Krislov hält die spieltheoretischen Annahmen zwar für ein übersimplifiziertes Modell, gleichwohl aber für eine sinnvolle Motiv-Isolation. Inwieweit kann aber die Maxime »Gib die entscheidende Stimme in einer möglichst kleinen Gewinnkoalition ab!« überhaupt sinnvolles richterliches Motiv sein? "Sinnvoll «, das soll sich bemessen an der Eigenart richterlicher Argumentation und der allgemeinen Justizfunktion. Dann läßt sich nicht mehr absehen von inbaltlicben Erwägungen und außergerichtlichen Macbtbeziebungen.

3. In beide Richtungen hat Schubert Erweiterungen vorgenommen, ohne aber das spieltheoretische Konzept in Frage zu stellen. Einmal behandelt er nämlich eine besondere Fallgruppe (Fälle, die unter den Federal Liability Act, FELA, fallen). Auf deren spezifische Konstellationen, Argumentationsmuster müssen richterliche Durchsetzungsstrategien bezogen werden. Diese selbst werden zudem revidiert: primärer Machtaspekt ist nun die Abnahme der Entscheidungen durch untere Gerichte ${ }^{5}$. Also: wie müssen Richter handeln, wenn sie ihre Ansichten in spezifischen Fällen bei Untergerichten durchsetzen wollen? Schubert hält vier Strategien des Supreme-Court-Richterblocks für möglich, der so viel wie möglich FELA-Fälle für den Kläger, den verletzten Arbeiter, entscheiden will.

I. Er könnte in allen Fällen eine Uberprüfung (scertioraric) gewähren, gleich ob der staatliche Arbeitgeber oder der Arbeiter vor dem SC klagt. Er könnte aber eine Prüfung verweigern, wenn bereits die unteren Gerichte gegen den Arbeitgeber entschieden haben.

2. Es könnten alle Anträge von Arbeitern zugelassen werden, keine der anderen Seite. Dann könnten aber auch solche Fälle darunter sein, in denen wegen der gesetzlichen Bindung - dic erkennt Schubert an - gegen den Arbeiter entschieden werden müßte, also ein unguter Präzedenzfall geschaffen würde.

3. Alle Arbeitgeber-Klagen könnten abgewiesen und nur solche Arbeiter-Anträge zugelassen werden, die erfolgversprechend sind: ssome evidence ${ }^{\theta}$ besitzen, 'most persuasives (ebd. S. 245) sind.

Schubert, N. I (1964) S. 425 .

- Schubert, N. I (1959), S. 23 r. 
4. Für die sicherste Strategie hält Schubert, wenn die betreffenden Richter keine Arbeitgeber-Anträge zuließen und nur solche des Verletzten, die in der ersten Instanz anerkannt, in der zweiten aber abgelehnt wurden.

Die Größe des Blocks wird dann selbst zum Moment der Uberzeugungsbildung: solange noch Stimmen hinzugewonnen werden müssen - also wenn die Gruppe nur aus drei oder vier Mitgliedern besteht -, sollten nur die >most persuasive claims of workers ${ }^{7}$ zur Entscheidung in der Sache zugelassen werden (bei einer Vierer-Gruppe gilt: w weed out the most frivolous cases (ebd.)). Aber selbst eine Majorität von fünf muß immer noch Rüdksicht nehmen: auf die öffentliche Meinung, Rechtszeitschriften, untere Gerichte, Mitglieder, die nur marginal zugehörig sind. Es wäre ein too redkless play‘, wenn alle Fälle akzeptiert würden.

Der ursprüngliche »Macht«-Begriff ist also aufgelöst. Es geht um die Durchsetzung politischer Anschauungen vornehmlich bei Kollegen und Untergerichten. Die Strategien nähern sich der faktischen Motivlage der Richter, ihren Plausibilitätserwägungen an. - In die Kalkulation der Durchsetzungschancen geht neben der Argumentation vom Fall her auch die Überlegung ein, die Kollegen und Untergerichte nicht zu verprellen: deshalb komme es nicht auf die absolut höchste Zahl von Gewinnen in besagten Fällen an, sondern lediglich auf »a consistent pattern of wins", einen möglichst hohen Prozentsatz von Gewinnen gegenüber Verlusten ${ }^{8}$.

Die spieltheoretischen Maximen gehen mit der Annäherung an faktisches richterliches Handeln in Klugheitsregeln über. Walter F. Murphy hat ein spieltheoretisches Modell zur Interpretation der richterlichen Durchsetzung politischer Ziele ausdrücklich abgelehnt: die richterlichen Motive seien gemischt ${ }^{\theta}$. Er formuliert lediglich einen lockeren Katalog von Strategien zur Erreichung politischer Vorstellungen.

Die Bedingungen der Koalitionsbildung in Entscheidungsgruppen bedürfen weiterer Spezifizierung. Sie können weder erfaßt werden durch von möglicher Motivation abstrahierende Machtmaximierungs-Strategien, nicht durch formale Abstimmungskohäsion ohne Bezug auf die entschiedenen Probleme ${ }^{10}$, noch durch

7 Schubert, N. I (1964), S. 426.

${ }^{8}$ Ebd., S. 425 - Zur Kritik dieses Macht-Begriffs s. Walter Berns, Law and Behavioral Science, in: Hans W. Baade, ed., Jurimetrics, New York: Basic Books, 1963, S. 185-212. Ein Entscheidungsverhältnis von $52:\{$ wäre nach Schubert günstiger als eines von $20: 4$.

- Walter F. Murphy, Elements of Judicial Strategy, Chicago: Univ. of Chicago Press, 1964 Politische Motive bei Richtern anzunehmen ist sinnvoll, weil Recht ( $r$ ) Mittel der Formalisierung von »publiy policy « sei; (2) Richter notwendig eine Rolle spielten beim »shaping public policy \& (nicht nur bei der Überprüfung von Gesetzen); (3) weil sie einen Entscheidungsspielraum durch ihre Wertpräferenzen ausfüllen könnten. Das Problem eines politisch bewußten Richters (das in einem unpolitischen Selbstverständnis allenfalls verdrängt werden kann) ist dann einmal, den aktuellen Entscheidungsspielraum zu ermitteln, der durch symbolische Schranken gezogen ist, aber auch durch das Legitimationspotential (z. B. »Hüter der Verfassung «, - Subsumptionsautomat*), das er nicht überziehen darf; und herauszufinden, wie er die Durchsetzung seiner Ziele in diesem Rahmen durch legitime Mittel erreichen kann. - Harold J. Spaeth [Judicial Power as a Variable Motivating Supreme Court Behavior, in: Midwest Journal of Political Science, 6 (1962), S. 54-82] untersucht, ob die Motivation richterlichen Handelns durch Vorstellungen über "true judicial power items « bestimmt sei. Die Schwierigkeit besteht darin, klare »power «-Fälle zu isolieren, in denen Machtüberlegungen sich nicht hinter juristisch-doktrinären UUberlegungen verschanzen. Er wählt dafür Fälle, in denen der Supreme Court Entscheidungen der Interstate Commerce Commission zu überprüfen hatte, wobei manche explizite Machtüberlegungen, die Rolle der Justiz im staatlichen System betreffend, enthalten; Fälle, in denen die kumulativen Skalen zur Messung ökonomischer Einstellungen inkonsistent sind und eine power-Skala mit den dominanten Variablen activism/restraint höhere Konsistenz erbringt; Fälle, in denen Fragen der Funktion der Bundesgerichtsbarkeit zumindest implizit angesprochen sind. Es läßt sich dann empirisch belegen, daß Richter Macht gegenüber anderen staatlichen Instanzen ausüben, die unverträglich ist mit einem unpolitischen Verständnis der Justiz.

10 Wie etwa in den Arbeiten von Charles Herman Pritchett: The Roosevelt Court: A Study in 
bloßen professionell-juristischen Konsens über doktrinäre Gehalte. Es kommen hinzu ideologische Affinitäten, das Selbstverständnis von der richterlichen Rolle11. - Der dynamische Aspekt jedoch bleibt dabei unberüdksichtigt: Blodkbildungen werden nicht als Resultat von Interaktionen ausgewiesen ${ }^{12}$. Snyder hatte zunächst historisch untersucht, welche Tendenzen in der Bildung von Blökken zu beobachten sind, worauf sie zurückzuführen sind ${ }^{13}$. Einen Wandel vollzogen weniger die einzelnen Richter in ihren Einstellungen (mit Ausnahme von Frankfurter und Jadkson), er wurde vielmehr für das Gericht insgesamt durch Neukommer ausgelöst, ohne daß ein klarer Zusammenhang der politischen Richtung, die der ernennende US-Präsident vertrat und dem politischen Trend des SC zu erkennen wäre. Auch der Versuch Ulmers mit systemtheoretisch verdünnten Kategorien Stabilitätsbedingungen in der SC-Interaktion zu finden, bleibt unbefriedigend ${ }^{14}$. Er ordnet jedem Richter nach dem Shapley-ShubikIndex eine relative "power-role $\approx$ zu und versucht herauszufinden, welche Faktoren zu einer Veränderung des Indexes im Laufe der Zeit führen ${ }^{15}$. Als besonderen Stabilitätsfaktor arbeitet er das Verhalten des Chief Justice heraus ${ }^{16}$.

Die spieltbeoretischen Macht-Strategien lösen sich also - auf richterliches Handeln angewendet - in Klugheitsregeln auf. Die Blockanalyse wird substantialisiert durch Momente inhaltlicher juristischer Erwägungen, richterliches Selbstverständnis (auch von der politischen Funktion der Justiz), ideologische Affinität, Gruppenrollen und besondere Verhaltensstile. Beide Male ist die Revision des zugrundeliegenden Motivationskonzepts ausschlaggebend für die Transformation der ursprünglichen Ansätze. Um den reinen Strategien der Spieltheorie ihren tautologischen Charakter zu nehmen, sie in die tatsächliche Motivation einzuklinken - ohne auf ceteris paribus-Klauseln zurüdkzugreifen ${ }^{17}$-, muß das Handlungsmodell selbst geändert werden.

Der spieltheoretische Ansatz hat zumindest auf das Phänomen der Interaktion 'von Subjekten hingewiesen, das in der verhaltenswissenschaftlichen Isolation des Aktors auf ein einsames Verhältnis zu seiner Attitüde nicht erscheinen konnte. Wie läßt sich die Interaktion zwischen Subjekten begreifen (für Richter:

Judicial Politics and Values, New York: Macmillan, 1948; Civil Liberties and the Vinson Court, Chicago: Univ. of Chicago Press, 1954. - Zur Kritik s. Harold J. Spaeth, Measuring Ideational Identity by Pairing Justices, in: PROD, I960, S. 22-23; S. Sidney Ulmer, Polar Classification of Supreme Court Justices, in: South Carolina Law Quarterly, 12 (1960), S. 407-417.

${ }^{11} \mathrm{~S}$. Sidney Ulmer, Toward a Theory of Sub-Group Formation in the United States Supreme Court, in: Journal of Politics, 27 (1965), S. $133-152$.

12 Walter F. Murphy, Courts as Small Groups, in: Harvard Law Review, 79 (1966), S. is6s1572 .

${ }^{13}$ Eloise C. Snyder, The Supreme Court as a Small Group, in: Social Forces, 36 (1958), S. $232-$ 238.

14 S. Sidney Ulmer, Homeostatic Tendencies in the United States Supreme Court, in: Ulmer, ed., Introductory Readings in Political Behavior, Chicago: Rand Mc Nally, 1961, S. 167-188.

15 Die Anwendung eines Gleichgewichtsmodells auf Interaktionen ist problematisch, solange keine Kriterien der Stabilität resp. der Dysfunktionalität angegeben werden können. Für die Stabilität von Interaktionen besagt die Stabilität der Rangfolge der spower-roles` nichts.

${ }^{10}$ David J. Danelski (The Influence of the Chief Justice in the Decisional Process, in: Murphy/ Pritchett, eds., Court, Judges, and Politics, New York: Random House, 196I, S. 497-508) unterscheidet mit Berufung auf Robert F. Bales (Interaction Process Analysis. A Method for the Study of Small Groups, Cambridge Mass.: Harvard Univ. Press, 1950 ) zwischen einer aufgabenorientierten Rolle des Vorsitzenden (task leadership) und einem Stil, der auf informelle Harmonie zielt (social leadership). Sie werden auf ihre Auswirkungen in der Gruppe hin untersucht: Konflikte und Kohäsion in ihr, ihre Zufriedenheit und Produktivität.

17 Vgl. Hans Albert, Marktsoziologie und Entscheidungslogik, Neuwied-Berlin: Luditerhand, 1967. Erving Goffman hat die Schritte systematisch aufgezeigt, die für die Transformation einer Spielsituation in alltägliches Handelns notwendig sind. Erving Goffman, Where the Action Is, in: Interaction Ritual, Garden City, New York: Anchor Books, 1967, S. 149-270. 
die in beratenden Gremien, im Prozeß selbst, nach außen hin)? Die Interaktion - das ist das gängigste Konzept - vollzieht sich in Rollen (wir hatten das an der besonderen Rolle des Chief Justice angedeutet), in relativer Erwartbarkeit der Reaktionen des anderen, ohne daß diese aber strikt kalkulierbar eingeschliffen wären. Wie verhalten sich dann die "persönlichen « Momente von reflexivem Selbstverständnis und spontaner Expressivität zu den rollenkonformen Verkrustungen im Verhalten? Schließlich: Handlungsmotive waren sinnvoll nicht ohne Rekurs auf »inhaltliche « Erwägungen zu interpretieren, d. h. nicht ohne Rückgriff auf die symbolischen Gehalte, in denen sie kommuniziert werden. Den Anspruch, Handlungen als symbolisch geleitete Rollenübernahme in Interaktionen zu konzeptualisieren - und es geht zunächst nur um ein theoretisches•Konzept -, versucht eine Richtung der amerikanischen Soziologie einzulösen: der symbolische Interaktionismus.

\section{Symbolischer Interaktionismus - Rollentheorie}

Die drei genannten Aspekte möchte ich vornehmlich an Arbeiten von George Herbert Mead, der als Stamnvater des symbolischen Interaktionismus gelten kann, knapp darstellen. ${ }^{18}$

r. Die Bedeutung von Symbolen für unser Handeln;

2. Das Verhältnis von einem Subjekt zum andern (ego - alter) in Interaktionen (Rollenübernahme);

3. Das Verhältnis des Handelnden zu seiner Rolle.

Ad I. In unserem Diskussionszusammenhang empfiehlt es sich, die Annahmen des symbolischen Interaktionismus nicht als selbständigen Block neben anderen zu diskutieren, sondern sie im Hinblick auf eine Kritik der verhaltenswissenschaftlichen Konzepte einzuführen. Mead selbst gewinnt seine Ansicht weitgehend in Konfrontation mit dem kruden Behaviorismus Watsonscher Provenienz. - Symbole - gemeint sind hier vornehmlich sprachliche - durchbrechen den Bogen, der sich zwischen einem Reiz und einer anpassenden Reaktion spannt. Die Verhaltensorientierung geschieht nicht mehr durch instinktgesicherte Umweltanpassung, sondern durch interne symbolische Verarbeitung von Informationen. Umwelt besteht nicht nur aus beobachtbaren physikalischen Stimuli, sondern auch aus vergegenständlichten und intersubjektiv geteilten Bedeutungen und Werten. Wir leben in einem "symbolic environment «. Damit ist die Bedeutung der Symbole als Medien einer aktiven Umweltinterpretation und -konstitution bezeichnet. $\mathrm{Da}$ die Stimuli immer schon symbolisch vermittelt sind, können wir uns ihnen gegenüber aufmerksam, selegierend und reflexiv (und nicht nur anpassend) verhalten. Symbole stellen Handlungsimpulse still. Sie nehmen den Reizen ihre kausale Kraft. Als Mittel der Handlungsorientierung erlauben sie die Vorstellung von Handlungsalternativen, die Speicherung von Erfahrungen und deren Aktualisierung in Entscheidungen. Als Normen dienen

${ }^{18}$ G. H. Mead, Mind, Self, and Society (1934) (dt. Obersetzung: Geist, Identität und Gesellschaft, Frankfurt a. M.: Suhrkamp, 1968); ders., The Philosophy of the Act, Chicago: Univ. of Chicago Press, 1938. Zwei deutsche Auswahlbände sind 1969 erschienen: Philosophie der Sozialität (Suhrkamp), Sozialpsychologie (Luctterhand). Zur allgemeinen Einführung in den Ansatz des symbol. Interaktionismus s. Arnold M. Rose, ed., Human Behavior and Social Processes, London: Routledge and Kegan Paul, 1962. S. auch Anselm Strauss, Spiegel und Masken. Die Suche nach Identität, Frankfurt a. M.: Suhrkamp, I 968 und Peter Berger und Thomas Ludkmann, Die gesellschaftliche Konstruktion der Wirklichkeit, Frankfurt a. M.: S. Fischer, 1966 (verbunden mit einem wissenssoziologischen Ansatz). 
sie der Stabilisierung von Erwartungen. Schließlich sichern sie einen durch Ambiguitäten, Innovationen und Abweichungen stets gefährdeten Konsens. Intersubjektivität konstituiert sich im symbolischen Äther.

Ad 2. Eine systematische Ableitung der Funktion von Symbolen vom Individuum und dessen einsamer Beziehung zur Umwelt her stößt allerdings auf das Problem der Begründung von Intersubjektivität. Es wird nicht dadurch gelöst, daß einem monologischen ego ein alter als Bestandteil der symbolischen Umgebung zugesellt wird. Mead wählt einen anderen Weg. Er geht nicht vom ego aus, sondern von einem »sozialen Prozeß «, einer "sozialen Handlung«, worin sich allererst identische Subjekte herausbilden. Es wird also vorgängig gesellschaftliche Verträglichkeit, die Reziprozität von Erwartungen, die mögliche Identität von Bedeutungen unterstellt - und dann erst nach den einzelnen Mechanismen dafür gefragt. Die wichtigsten sind ssignifikante Symbolee, Rollenübernabme und der sgeneralisierte Andere*. Eine Geste, bei Mead das erste Element von Intersubjektivität, ist nicht so sehr Ausdrudk eines substantiellen "Inneren «, vielmehr ein $"$ Reiz, der die Reaktion des anderen beeinflußt ${ }^{10}$. $"$ Sie sind Teil der Organisation der gesellschaftlichen Handlung. « (ebd.S. 83) Gesten sind dann signifikant, wenn sie im einen Individuum die gleiche "Haltung « sich gegenüber auslösen wie im anderen. Besser geeignet für die wechselseitige "Anpassung " von Reaktionen als leibnahe Gesten sind sprachliche Symibole. Als vokale Gesten drücken sie nicht bloß eine "Idee « aus und produzieren beim anderen diese Idee, sie ermöglichen zugleich Selbstkontrolle und damit erst, daß wir auf den eigenen, vergegenständlichten Reiz implizit so reagieren, wie der andere es explizit tut. Die Reaktion kann als Reiz für die Kontrolle der eigenen Handlungen dienen; sie kann aber auch schon symbolisch antizipiert, gedacht werden. Die ${ }^{\text {Bedeu- }}$ tung « des Symbols ist die Reaktion, die ihm folgt. Der "Sinn « liegt gleichsam $z$ wischen Geste und konsequenter Handlung. »Der Sinn des von uns Gesagten ist die Tendenz, darauf zu reagieren." (ebd. S. 106) "Der Sinn an sich, d. h. der Gegenstand des Denkens, entsteht in der Erfahrung dadurch, daß sich der einzelne dazu anregt, die Haltung des anderen in seiner Reaktion auf das Objekt $\mathrm{zu}$ übernehmen. Sinn ist das, was anderen aufgezeigt werden kann, während es durch den gleichen Prozeß auch dem aufzeigenden Individuum aufgezeigt wird. Insoweit der einzelne ihn sich selbst in der Rolle des anderen aufzeigt, macht er sich dessen Perspektive zu eigen, und da er ihn dem anderen aus seiner eigenen Perspektive aufzeigt, das Aufgezeigte also identisch ist, muß es in verschiedenen Perspektiven auftreten können. Es muß somit universal sein, zumindest in der Gleichartigkeit, die zu den verschiedenen Perspektiven gehört, die in der einzelnen Perspektive organisiert sind. (ebd. S. x29) Unsere Handlungsorientierungen gewinnen wir nicht nur situativ, aus der aktuellen Beziehung von ego zu alter. Das Phänomen der Generalisierung von Erwartungen über Personen und Situationen hinaus (z. B. Rechtsnormen mit allgemeinem Anspruch) versucht Mead mit dem Konzept des "generalized other « zu erfassen. Der generalisierte andere ist die personalisierte Bezugsinstanz sozial geltender Erwartungen. Im Begriff der Bezugsgruppe hat die soziologische Forschung das Moment der Verallgemeinerung von Handlungsperspeltiven zu sozialen Strukturen festgehalten, zugleich aber deren gruppenspezifische Ausrichtung vermerkt.

Identifizierbare Subjekte, die ihre Erwartungen in »sinnvolle«, d. h. für ego und alter identische Symbole kleiden, sind berechenbar. Rollenübernahme ist notwendige Bedingung der Voraussehbarkeit von Handlungen, also der Erwartungs-

10 G. H. Mead, Geist, Identität und Gesellschaft (N. I8), S. 8 I. 
stabilität. "Role taking is anticipatory behavior. «º Die Betonung des prognostischen Aspekts, der die Rollentheorie durchzieht, droht aber die ursprüngliche Einsicht in die intersubjektive Konstitution von Sinn zu verlieren. Dieser Prozeß wird stillgestellt, Akteure zu definitiven Rollenträgern verdinglicht. Es bleibt ein monologisches Handlungskonzcpt übrig, in dem Subjekte nur als Berechnungspartikel erscheinen. Letztlich sind ego und alter in formaler Reziprozitär der Rollenübernahme austauschbar. Bedeutungen, Wertorientierungen werden aus Kommunikationszusammenhängen herausgenommen; Begriffe haben dann keine Geschichte mehr, allenfalls noch einen Kern $\alpha$ und einen verschwommeren „Hof «. In diesen Metaphern gerinnen Bedeutungsambiguitäten, losgelöst von ihrer Basis: der Ambiguität von Perspektiven, die nicht vollständig verschmelzen.

Ad 3. Wie ist aber das Moment von Unverwechselbarkeit zu denken: daß ich mich als unvertretbare Identität gegenüber anderen Identitäten festhalten kann? Mead $f_{a} B t$ den Doppelaspekt von Identität - daß sie sich durch Ubernahme von Erwartungen anderer und durch unverwechselbare Akrivität des Subjekts konstituiert - mit den Begriffen von $*$ me* und $* I$ I . Dieses macht die spontane, niche erwartbare, schöpferische Seite aus. Daß Personen nicht vollständig in Rollen, den Erwartungen, die an sie in bestimmten Positionen geknüpft werden, aufgehen, ist in zahlreichen Aspeksen herausgearbeiter worden. (a) Die übliche Trennung von "role obligation « und *role performance" bezeichnet das Problem: die Definition der Rolle fällt nicht mit ihrer individuelien Interpretation zusammen. Rollen können "persönlich" moduliert werden, manche erfordern sogar »persönliches Verhalten (von Ärzten z. B.). Im Wortspiel »role taking * - »role making « ist das ausgedrüdkt ${ }^{21}$. Die Motive fallen nidht mit den Rollenerwartungen zusammen. (b) Es läßt sich die Annahme einer Reziprozität von ego und alter als vorweg konformistisch bestreiten. Nicht so sehr, daß Mißverständnisse, Konflikte, enttäuschende Abweichungen auftauchen, die dann allein als Reziprozitätsstörungen erfaßt werden können, wäre der Einwand, sondern daß die schillernde Ambivalenz und Gebrochenheit von Kommunikationen den Ausgangspunkt bilden müßte, von denen sich rabiat agonale - und darin reziproke - wie scheinfreundlich verkleisterte Interaktionen abheben lieBen. (c) Mit der Trennung von innen-außen läßt sich das Moment der Selbstdarstellung einführen. Wir identifizieren uns nicht nur mit anderen Rollen, wir werden auch als Personen identiniziert (identification with/of): durch Mienen, Gesten, leibnahe Ausdrudiswcisen, die unserer bewußten Kontrolle nicht vollständig zugänglich sind. Die Unterscheidungen von verbal discourse/appearance, Inhalts- und Beziehungsaspekt treffen diesen Punkt. (Daraus ergibt sich eine prinzipielle Asymmetrie in Kommunikationen: der andere verfügt angesichts unserer expressiven Darstellung über mehr Informationen, als dem Darstellenden zugänglich sind. Diese Asymmetrie kann durch Beziehen einer Beobachterrolle fixiert werden [bei Prüfern, Reportern, Richtern] oder durch eigenes Ausdrucksverhalten balanciert werden.) (d) War mit den Punkten (a) und (c) das Element spontaner Selbstdarstellung bezeichnet, so meint der Begriff der Rollendistanz die reflexive Vergegenständlichung des Rollenhandelns, die Signalisierung, da $B$ der Handelnde nicht vollständig in der Rolle aufgehen will, sich den Rollenanforderungen dann aber auch nicht total entzieht. Die Möglichkeit

20 Sheidon Stryker, Conditions of Accurate Role-Taking: A Test of Mead's Theory, in: Rose, ed., (N. I8), S. 46.

21 Ralph H. Turner, Role-Taking: Process Versus Conformity, in: Rose, ed., (N. I8), S. $20-40$. 
zu distanziertem Verhalten (durch Witz, Ironie, Parodie, Grimassen, Schnoddrigkeit etc. signalisiert) hängt sicher von der lebensgeschichtlich variierenden und schichtenspezifischen Ausbildung kommunikativer Fähigkeiten ab, zum anderen aber auch von dem Grad der Ernsthaftigkeit und Sanktionsgewalt, die mir Rollenerwartungen verbunden sind. Rollendistanz weist den Weg zwischen regungsloser Verweigerung von Rollenübernahme und dem Zerfließen in Rollen oder dem Zersplittern in Rollensegmenten, das dem Ich nur die spassive Phantasie unausgefüllter Räume«beläßr.

Die vorliegenden amerikanischen Untersuchungen, die das Rollenkonzept auf richterliches Handeln anwenden, holen die Komplexität des theoretischen Ansatzes nicht ein. Ich gehe deshalb nicht näher auf sie ein, referiere lediglich Grundgedanken². Im ersten Teil (KJ I970, S. 295 f.) fand bereits das Rollenkonzept in den Arbeiten Th. Bedkers Erwähnung. Er unterschied zwischen einer persönlichen Orientierung und einer an precedents. »Rolle» war aber bei ihm als "Wertorientierung " eines einsamen Subjekts konzipiert, die sich von Attitüden lediglich dadurch unterschied, daß in ihr das richterliche Selbstverständnis berüdksichtigt wurde. Ebenso meint Grossman mit Rolle lediglich deren subjektive Interpretation (»a consistent pattern of behavior on the part of an inividual in response to his conceprion of the nature of his function in a system (1) $)^{33}$ Jaros und Mendelsohn gehen vom Problem der unterschiedlichen Urteilspraxis aus. Sie wollen prüfen, ob die professionellen Rollenerwartungen die richterliche Tätigkeit beeinflussen oder ob persönliche Faktoren bedeursam sind. Sie kommen, wie Green (s. Teil r, S. 298 f.), zu dem Schluß, daß Richrer »in accordance with legally justifiable criteria " handeln ${ }^{24}$. Zu diesen legalen Kriterien zählen sie nicht nur Eigenschaften des Falles (Vorstrafen, „Schwere“; irrelevant seien Geschlecht, Rasse, Alter), sondern auch - da die richterliche Rolle nicht nur durch Pflichten gekennzeichnet sei, sondern auch durch Rechte: nämlich die Erwartung von Respekt - das Verhalten des Angeklagten vor Gericht. Die Art der Kleidung korreliert, außer bei ganz schweren Fällen, signifikant mit der Höhe der Strafe. Der von den Autoren entwickelte "Demeanour Index (kein Titel bei der Anrede des Richters, ausdrüdkliche Nichtübereinstimmung mit Feststellungen des Richters, Intonation, Sarkasmus, Herabsetzung von Gericht, Recht und Polizei, keine Reue) konnte nicht überprüft werden, da sich die meisten Angeklagten wanständig « benahmen. Jaros und Mendelsohn sind die einzigen Autoren, die gerichtliche Interaktionen auf diese Weise einbeziehen; zudem erkennen sie durchaus, daß die Rollendefinition abhängig ist von der jeweiligen Bezugsgruppe: ein Richter in den Südstaaten hat andere Erwartungen zu erfüllen (welche, verraten sie nichr). - Seidman macht klar, daß der Sinn von Regeln,

2- Theodore L. Bedker, Political Bchavioralism and Modern Jurisprudence, Chicago: Rand Mc Nally, 1964; Joel B. Grossman, Role-Playing and the Analysis of Judicial Behavior: The Case of Justice Frankfureer, in: Journal of Public Iaw, II (1962), S. 285-309; ders., Dissenting Blocs in the Warren Court: A Study in Judicial Role Behavior, in: Journal of Politics, 30 (1968), S. 1068-1090; Dororhy James, Role Theory and the Supreme Court, in: Journal of Politics, 30 (1968), S. I60-1 \$6; Dean Jaros und Robert J. Mendelsohn, The Judicial Role and Sentencin: Behavior, in: Midwest Journal of Pol. Science, I I (1967), S. 471-488; Robert B. Seidman, The Judicial Process Reconsidered in the Light of Role-Theory, in: Modern Law Review, 32 (1969), S. 516-531. - S. auch: Rüdiger Lautmann, Rolle und Entscheidung des Richters - Ein soziologischer Problemkatiog, in: LautmanniSchelsky/Maihofer (N. 4 ), S. $38 \mathrm{I}-4 \mathrm{I} 6$.

23 Grossman, Nr. 22 (1962), S. 294.

24 Jaros/Mendelsohn, N. 22, S. 475. Sie entgehen nicht der bei Green im ersten Teil schon bezeichneten Shwierigkeit, daß sie nicht unabhängig von der richterlichen Verwendung, die durch Vorurteile bestımme sein kann, die Kriterıen bestimmen können. sollengemäß handein heiße dann nur noch, daB juristische Argumente verwendet werden. 
die explizit angewendet werden und diese Anwendung leiten, in klaren und problematischen Fällen abhängig ist von dessen intersubjektiver Bestimmung: dem *common agreement among law trained professionals« (Seidman, S. 522). Sie sind die relevante Bezugsgruppe zur Bestimmung des linguistischen Gehalts. Er kritisiert das unpolitische Selbstverständnis dieser Gruppe, die sich hinter einer *communis opinio doctorum « verschanzt, die Regeln, die an klaren Fällen entwickclt wurden, auf problematische anwendet, um den Schein von Neutralität zu wahren. Seidman verfällt aber eben dieser Begriffsverdinglichung, wenn er vorschlägt, daß Regeln zur Lösung von "trouble cases \& formuliert werden müßren - als sei die $\gg$ Problematik « eines Falles dessen dingliche Eigenschaft und nicht Resultat eines intersubjektiven Konflikts von Perspektiven, der nur zum Schein und $z$ wangshaft stiligestellt wird.

Offen geblieben ist nach der bisherigen Diskussion

- wie der Begriff der Macht sinnvoll auf die Justiz angewendet werden kann;

- welche Bedeutung Symbolen für richterliches Handeln zukommt;

- wie sich die gerichtliche Interaktion begreifen läßt.

Niklas Lubmann hat diese Fragen im ersten Teil seines Buches Legitimation durch Verfabren (Neuwied-Berlin: Luchterhand, 1969) behandelt. Ich wende mich deshalb einer intensiven Diskussion dieser Veröffentlichung zu.

\section{Macht}

a) Das Bezugsproblem, unter dem Luhmann die Funktion rechclich geregelter Verfahren (Gerichtsverfahren, politische Wahl, parlamentarische Gesetzgebung, Entscheidungsprozesse der Verwaltung) analysiert, ist das der Abnabme von Entscheidungen. Verfahren - und im folgenden beschränke ich mich auf gerichtliche - sollen wahre und gerechte Urteile sichern. Zugleich aber unterstehen sie der Notwendigkeit rechtskräftigen Entscheidens. Wic ist dieser Widerspruch in der offiziellen Legitimation zu erklären? Gewöhnlich behilf man sich dadurch, daß der Wahrheitsbegriff relativiert, Rechtssicherheit als Element der Gerechtigkeit deklariert wird. Luhmann fragt radikaler, ob die Garantie von Wahrheit überhaupt Ziel von Verfahren sein kann. Wenn es nämlich deren Funktion ist, andere zu motivieren, die Entscheidung als eigene Verhaltensprämisse zu übernehmen, so könnte diese Ubbertragung auch durch andere Mechanismen als den der Wahrheit übernommen werden. Systemtheoretisch leistet "Wahrheit im sozialen Verkehr die Ubertragung reduzierter Komplexität. Wahrheit ist Vorstellungsübertragung auf Grund intersubjektiv zwingender Gewißheit. Vorstellungen werden hier nicht übernommen auf Grund von persönlicher Sympathie (Liebe), von Mitgliedschaft oder Machtunterlegenheit. Das Engagement für Wahrheit »bezieht sich nicht mehr auf die konkrete Individuaiität des Menschen (wie etwa Liebe) und nicht mehr auf seinen sozialen Status oder seine Rollen (wie etwa Mitgliedschaften) und nicht mehr auf sein Durchsetzungsvermögen (wie etwa Macht), sondern auf seine Subjektivität. In Wahrheitsfragen ist der Mensch als Subjekt engagiert, das heißt als jemand, der als Träger des Sinns der Welt mit in Betracht kommt (bzw., wenn er Wahrheiten ableugnet, nicht mehr in Betracht komme).« (S. 24) - Neben Wabrheit als evidentem Ubertragungsmechanismus kann auch der der Macht treten; und er muB es, weil angesichts der Wissenschaftsentwicklung immer weniger wahrheitsfähig geworden ist. "So gesehen ist das Ziel rechtlich geregelter Verfahren, Reduktion von Komplexitär 
intersubjektiv übertragbar zu machen - sei es mit Hilfe von Wahrheie, sei es durch Bildung legitimer Macht zur Entscheidung.* (S. 26)

b) Das Problem der Legitimität von Macht lautet herkömmlich: $*$ Wie ist es möglich, wenn nur wenige entscheiden, die fakrische Uberzeugung von der Richtigkeir oder der verbindlichen Kraft dieses Entscheidens zu verbreiten? \& (S. 27) Die übliche Antwort lautet: durch physischen $Z$ wang oder Konsens. Luhmann geht dagegen rom Phänomen des faktischen, morivlosen Akzeptierens aus. Legitimität faßt er auf als meine generalisierte Bereitschaft, inhaltlich noch unbestimmte Entscheidungen innerhalb gewißer Toleranzgrenzen hinzunehmen « (S. 28). •Komplexe moderne Gesellschaften* erforderten gerade eine Generalisierung des Anerkennens: $d$. h. es komme auf ein motivfreies, von den Eigenarten individueller Persönlichkeiten unabhängiges Akzeptieren an, das ohne allzu viel konkrete Informationen typisch voraussehbar ist. Es kommt auf bloß faktisches Anerkennen an, das Entscheidungen nicht mehr an Prinzipien eines richrigen Lebens bemißt.

c) Mir den Begriffen des Anerkennens und Lernens versucht Luhmann das Problem der Legitimität - als faktische Hinnahme von Entscheidungen und Entscheidungsprämissen - zu spezifizieren. Wenn wir fremde Entscheidungen akzeptieren, bauen wir in unsere identisch bleibende Persönlichkeit neue Erwartungsstrukturen ein. Wir versuchen unsere neue Umwelt mit uns zu harmonisieren. Nicht nur auf den Prozeß der Ubernahme fremder Entscheidungen, auch allgemein auf den der Verarbeitung von Enttäuschungen bezieht Luhmann den Begriff des Lernens. Er bezeichnet die wirksame Einstellungsänderung, die Anpassung an eine vormals frustrierende Umwelt: wenn wir lernen, finden wir uns ab, protestieren nicht. Demnach geht es bei der Legitimation von Entscheidungen im Grund um ein effektives, möglichst störungsfreies Lernen im sozialen System. * (S. 35) Solches Lernen kann nicht vom einzelnen allein geleistet werden; es bedarf sozialer Stützen. sLegitimität beruht somit gerade nicht auf sfrei-williger Anerkennung, auf persönlich zu verantwortender Uberzeugung, sondern im Gegenteil auf einem sozialen Klima, das die Anerkennung verbindlicher Entscheidungen als Selbstverständlichkeit institutionalisiert und sie niche als Folge einer persönlichen Entscheidung, sondern als Folge der Geltung der amtlichen Entscheidung ansieht ... Nur wenn man die Bindung des Legitimitätsbegriffs an die persönlich geglaubte Richtigkeit der Entscheidungen aufgibr, kann man die sozialen Bedingungen der Institutionalisierung von Legitimität und Lernfähigkeit im sozialen System angemessen untersuchen.* (S. 34)

\section{Symbolische Implikation versus Verfabren}

Luhmann unterscheidet zwischen einer Legitimation durch Verfabren und der Legitimation durch sprachlich-symbolische Implikation. Diese Art der Rechtfertigung erbringen Juristen in ihren Entscheidungsbegründungen; sie legitimieren im Medium juristischer Dogmatik durch plausible, glaubwürdige Darstellung der Entscheidungsmotive, die noch Werten der Gerechtigkeit verpilichtet sind. Bei der Legitimation durch Verfahren dagegen sgeht es um die Umstrukturierung des Erwartens durch den faltischen Kommunikationsprozeß, der nach Maßgabe rechtlicher Regelungen abläuft, also um wirkliches Geschehen und niche um eine normative Sinnbeziehung. * (S. 37) Dieser Unterscheidung liegt also die von Sollen und Sein zugrunde. 
Die juristischen Regeln, materielle und formelle, strukturieren Verfalıren nicht vollständig. Prozesse sind auch nicht bloße Rituale: der Ausgang muß als ungewiß erscheinen. Sie besitzen eine cigene Verfahrensgeschichte, die durch Ubernahme von Verfabrensrollen durch die Beteiligten nach und nach aufgebaut wird. Dadurch legen sich die Betciligten selbst fest. Was sie bei der nemeinsamen Wahrheitssuche « trotz kontradilitorischer Interessen zusammenhält, zum Mitspielen motiviert, ist neben der Gewißheit, daß eine Entscheidung ergehen wird, gerade die Ungewißheit des Ausgangs. Anwältc zeigen sich besonders kooperationswillig, nicht nur wegen ihres Standesethos, sondern weil sie öfters mit demselben Gericht zu tun haben, mit dem sie es nicht verderben wollen sowohl in Verhandlungen nicht, wie auch bei informellen Kontakten.

a) Die Verstrickung der Beteiligten in die Verfahrensgeschichte ist der erste von Luhmann angeführte Mechanismus, der zur Abnahme von Entscheidungen (also zur Lösung des Bezugsproblems) verhilt. Daß im Verfahren die Selekrions- und Beurteilungskriterien erst erarbeitet, »konkretisiert « werden müssen, zugleich eine Entscheidung »nach Rege!n « zu erwarten steht, eröffnet den Beteiligten die Möglichkeit zu alternativenreichem Handeln, motiviert sie zu selbständiger Risikoübernahme, um die Ungewißheit des Ausgangs in ihrem Sinn zu reduzieren. Sie schaffen sich in einer offenen Situation eigene Verbindlichkeit. *Als Zukunttsplan absorbiert das Verfalıren Ungewißheit, als Geschichte wird es Bindung.* (S. 94) Wer in Verfahrensrollen mitspielt bleibe nicht einsamer Spieler. Rollen gewinnen allererst ihren Sinn aus ihrer komplementären Funktion: sie enthalten eine Aufforderung, sich korrespondierend zu verhalten und ebenso signalisieren sie die Bereitschaft, Erwartungen des anderen zu treffen. $\rightarrow$ Das Rollenverhalten führt mithin zu einer Einfühlung in die Rolle des anderen, zu einem Austausch von Perspektiven, zur Stabilisierung komplementärer Verhaltenserwartungen und übergreifender Prämissen sinnhaften Verhaltens - oder es muß mangels Verständigungsmögliçkeit abgebrochen werden. “ (S. 86)

Es ist nun die Frage, ob schon durch den Mechanismus der rollenmäßigen Verstrickung (commitment) in die Verfahrensgeschichte Entscheidungen leidhter abgenommen werden. *Vermutlich ist dies die heimliche Theorie des Verfahrens: daß man durch Verstrickung in ein Rollenspiel die Persönlichkeit einfangen, umbilden und zur Hinnahme von Entscheidungen motivieren könne.< (S. 87) Wenn es auch empirische Belege dafür gibr, daß durch die Beteiligung am Entscheidungsprozeß die resultierenden Entsdheidungen eher abgenommen werden, so bleibt gleichwohl das Faktum, daß „Beteiligte « unzufrieden oder empört den Gerichtssaal verlassen. Um diesen im Verfahren selbst nicht reduzierten Uberschuß an Protest problemlos zu halten, gibt es wcitere Mittel; ja, es scheint schließlich bei Luhmann elier so, daß dem Verfahren selbst wenig Bedeutung für die Milderung von Enttäuschungen zukommt. *Verfahren erzeugen nicht nur bleibende Einsichten, sondern auch bleibende Enttäuschungen. Ihre Funktion liegt nicht in der Verhinderung von Enttäuschungen, sondern darin, unvermeidbare Enttäuschungen in die Endform eines diffus verbreiteten, privaten Ressentiments zu bringen, das nicht Institution werden kann.« (S. I1 2)

b) Es müssen also weitere Mechanismen zur Verfügung stehen, die nach dem Verfahren die Verarbeitung von Enttäuschungen erlauben - -Verarbeitung « für das soziale System, nicht für den einzelnen: der wird mit seinen Enttäuschungen allein gelassen. Die Privintisierung von Protest gelingt einmal dadurch, daß dem Enträuschten vorgehalten werden kann, er habe, weil er sich als Mitspielez ver- 
stricken ließ, dic Rechtsordnung anerkannt. Nach Ableistung der Arbeit an der Verfahrensgeschidite "finder er sich wieder als jemand, der die Normen in ihrer Geltung und die Entscheidenden in ihrem Amte bestätigt und sich selbst die Möglichkeit genommen hat, seine Intercssen als konsensfähig zu generalisieren und größere soziale oder politische Allianzen für seine Ziele zu bilden. Er hat sich selbst isoliert.« (S. 117) Protest wäre nur um den Preis von Selbstwiderspruch möglich.

Der Begriff individuell zurechenbarer Schuld dient den Hütern der Normalität als Enttäuschungscrklärunga ${ }^{23}$. Es ist ein Individuum, nicht kausale Umstände, Mitmenschen, organisatorische, institutionelle Bedingungen, dem die Normverletzung zugerechnet werden kann. Wer protestiert, wird zum einzelnen Zurechnungspunkt zusammengezogen; für den, der als zurechnungsunfähig angesehen wird, stchen andere geschlossene Anstalten bereit; wer das juristisch-individualisicrende Zurechnungsspiel ïberhaupt negiert, bekommt es mit dem objektiven Geist einer strafenden Gesellschaft zu tun: er fällt durch ihre von Juristen behürete Plausibilitärsstruktur hindurch.

Vereinzelung wird schließlich dadurch erreicht, daß der Urteilende sich in ein anonymes Geflecht der "Rechtsordnung « zurückziehen kann, mit der der Protestierende dann unmittelbar konfrontiert wird. Es war nicht der Richter X, der verurteilt hat, er hat nur die gesetzlich vorgesehene Rechrsfolge ausgesprochen. Die Vorstellung von Rechrsanwendung als unpersönliche Subsumption übernimmt Luhmann im Begriff der $*$ konditionalen Programmierunga. Hierbei werden definierte Informationen aus der Umwelt (Tatbestände) »unparteilich « mit vorgeschriebenen Ausgaben des Entscheidungssystems (Rechtsfolgen) verknüpft. Kritik ist dann nicht an Folgen des Urteils möglich, sondern nur an der immanenten Richrigkeit des „Anwendungs «-Prozesses. Der Richter kann allenfalls gewisser Vorurteile verdächtigt werden, von Folgeverantwortung ist er entlastet.

Meine Kritik entfalte ich in fünf Punkten:

I. Auf Luhmanns Wabrheitsbegriff gehe ich nur kurz ein.

2. Das Lernkonzept und

3. das der Legitimität werden auf ihre gegenaufklärerischen Gehalte hin untersucht (Anpassungsverhalten und ontologische $»$ Faktisierung «).

4. An der Differenz von "symbolischer Implikation « und "Verfahren« werde ich zu zeigen versucien, inwieweit Luhmann ohne normativen Sinnbezug auskommen kann.

5. Schließlich möchte ich gegen Luhmanns Legitimation von Verfahren durch Entproblematisierung der gerichtlichen Interaktion diese als eine systematisch verzerrte Kommunikation darstellen.

I. An Luhmanns Wahrheitsbegriff ist für unsere Diskussion einmal relevant, daß die funktionale Hinterfragung von "Wahrheit a auf ihre Ersetzbarkeit durch andere Mechanismen es nicht mehr erlaubt, inhaltliche Kriterien für Wahrheit selbst zu formulieren. Als einzige Idealisierung bleiben das Engagement des Menschen als »Subjekt« und dessen Evidenzerlebnisse ${ }^{28}$. Festzuhalten ist weiter-

23 Niklas Luhmann, Normen in soziologischer Perspektive, in: Soziale Welt, 20 (1969), S. 28$4^{8}$ (S. $38 \mathrm{f} ., 45 \mathrm{f}$ ). Auf den Schuld-Begriff greift Luhmann in Iegitimation durch Verfahren. niche ausdrücklich zurüds.

2s Eine ausführliche Diskussion des Luhmannschen $\mathbb{W}_{2 h}$ heitsbegriffs, insbesondere der Schwierigkeiren, die sich aus der Anwendung des Begrifs auf die eigene Theorie ergeben, finder sich bei Jürgen Habermas, Theorie der Gesellschaft oder Sozialtechnologic. Eine Auseinanderserzung mit Niklas Luhmann, Manuskript, S. 107 ff. 
hin, daß die Obertragung von *Wahrheit« ohne Begründungen auskommen soll: sic ist »subjektivistisch « evident. Begründungsprobleme dagegen scheinen Indiz für Machtstrukturen zu scin: sie müssen legitimiert werden.

2. Durch Legitimation sollen Entschcidungen weniger für viele andere abnehmbar gemacht werden. Weil es dabei vornchmlich um Enescheidungen geht, die den eigenen Motivationen nicht entsprechen, besteht das Problem in der Obernahme mißliebiger Verhaltensprämissen. Dieses Problem spezifiziert Luhmann zunächst lerntheoretisch.

a) »Lernen* ist für ihn ein Mechanismus der Enttäuschungsverarbeitung. Wir können Enträuschungen überwinden einmal dadurch, daß wir das enträuschende Ereignis verurteilen, abschieben, übergehen etc., da $B$ wir unsere Erwartungen stabil halten: wir lernen dann nicht, sondern setzen unsere alte Perspektive durch. Wir können aber auch unsere Erwartungsstruktur auf ein enttäuschendes Ereignis hin ändern, sie veränderten Gegebenheiten anpassen. Einmal verhalten wir uns also dogmatisch-normativ (wie es in der Rolle des Richters am klarsten ausgeprägt ist), zum anderen kognitiv, lernbereit (wie in der Rolle des Wissensdiaftlers, der sich über jede Falsifikation freuen sollte) ${ }^{27}$.

Für Luhmann lernt der, der enttäuschende Ereignisse durch Wandel seiner Erwartungen verarbeiten kann. Dieses begrenzte Lernkonzept, die Bindung des Lernbegriffs an den der Enträuschung, hat zwar die Annahme für sich, daß Lernen ein schmerzhafter Prozeß ist. Ein Einstellungswandel vollzieht sich aber nicht nur aufgrund von Frustrationen, ebenso nämlich auch durch Belohnungen, -incentivesк, Reinforcement, Nachahmung und Identifikation mit einem (geliebten) Objekt. Für die Lerntheorie heißt "Lernen« denn auch nur allgemein, daß eine Veränderung im Zusammenhang von Reiz und Raktion stattgefunden, daß sich eine feste neue Verbindung, eine Umstrukturierung auch der Bewußtseinsformen ergeben hat.

b) Iuhmann geht, wie die Lerntheorien überhaupt, vom Stimulus-ResponseModell aus ${ }^{28}$. Enttäuschungen stoßen als Umweltreize auf eine mehr oder weniger flexible Erwartungsstruktur und haben Erwartungs- und Verhaltensänderungen

97 S. Luhmann, Normen (N. 25), S. ${ }_{4}$ ff. - Zur Lerncheorie verweise ich an deutschsprachigen Texten auf: Klaus Foppa, Lernen, Gedïhtnis, Verhalten. Ergebnisse und Probleme der Lernpsychologie, Köln-Berlin: Kiepenheuer \& Witsch, 2965; Franz Josef Stendenbach, Soziale Interakcion und Lemprozesse, Köln-Berlin: Kiepenheuer \& Wirsch, 19672; Franz Weinerr, Hg. Pädagogische Psychologie, Köln-Berlin: Kiepenheuer \& Witsch, 1967; Hans J. Hummel, Psychologische Ansz̈cze zu einer Theorie sozialen Verhaltens, in: René König, Ho., Handbuch der Empirischen Sozialforschung, Bd. II, Sturtgart: Enke, 1969, S. I I57-I 277, mit einer ausführlichen Bibliographie.

:9 Wir kommen hier von einer anderen Perspekrive auf das im ersten Teil (bes. S. 292) bereirs diskutierte verhaltenswissenschaftliche S-R-Modell zurüds. Es diente den Atrituidenforschern als Schema der Verbindung von Fall und Entscherdung. Das Argument war einmal, daß die Stimuli allererst aus den Responses gewonnen wurden (S-R-Zirkel) und daß mit dem S-R-Modell überhaupt en falsches Handlungsmodeil verwendet wird, weil mit ihm die Problematik des hermeneurischen Zirkels verdedk wird. Daß die hermeneurische Perspekcive sich dennoch zeigt, läßt sidr gerade am Attitüdenbegriff zeigen, der bereits eine Aufweichung des klassischen Behaviorismus darstellt: insofern als niche mehr Bedürfnisse, Triebe eines Organismus das Bindegiied von Reiz und Reaktion bilden, sondern Atrı̈uden ais Wertorientierungen. Damit ergibe sich das aice Problem, ob diese Konversionspattern (Bewußcscin, Meinungen, Erwartungen, Wertorientierungen etc.) noch in einer sphysikalistischen d. h. äußerlichen Beobachtungssprache gefabt werden können, oder ob nicht doch wieder auf das ehemals verfemte $\rightarrow$ mentalistische e, introspekcive Vokabular zurüdkgegriffen werden muß. Diese, gerade bei Schubert mangels theoretischer Reflexion uneingestandene Shwierigkest zeigt sich einmal darin, daß Stimulusund Attitüden-Kategorien verschmelzen (als Indiz des hermeneutischen Zirkels) und daß der Zugang zu den durchweg verbaien Daten (Urceilen) als subjektiv an den Problemrand geschoben wurde. Daß bei der Fallauswahl und der Definition der Skalen-Dimenstonen hermeneutische Kategorien allemal eingingen, wurde im gewaltigen Operationalisierungsnebel kumulativer Skalen verborgen. 
(*Lernen*) oder deren Aufrectrerhaltung zur Folge. Lernen ist für Luhmann eine Anpassungssreaktion an und auf enträusdiende Stimuli. Damit dient das S-R-Modell nicht bloB ( $\mathrm{I}$ ) als Schema der Zergliederung von Handlungssequenzen, der zeitlichen Unterscheidbarkeit von skonsequenten * Zuständen; audh nicht (2) als kausales Schema mit Erklärungswert, in dem Stimuli als unabhä̈gige Variable - diskriminierbare Eigenschaften von Objekten, Elemente der Klasse von Objekten, in deren Gegenwart der Organismus regeimäßig identifizierbare Reaktionen zeigt -, Reaktionen als abhängige Variable. Es wird vielmehr ein spezifisches Handlungs-Paradigma (3) mit dem S-R-Modell verbunden: das eines Organismus, der als $\gg$ Rezeptor $"$ Umweitreize passiv aufnimmt und adaptiv darauf, als "Effektor «, reagiert. Stimuli aktivieren allererst einen rezeptiven Organismus, lösen Reaktionen aus. Das S-R-Modell der herkömmlichen Lerntheorien geht von Anpassungsverhalten aus.

Daß Luhmann zur Diskussion des Bezugsproblems "Enttäuschungsverarbeitung * einen lerntheoretischen Ansatz verwender, der von Anpassungsreaktionen ausgeht - das macht das Ideologische aus: der lernt, der Enttäuschungen möglichst protestlos hirnimmt, den Enträuschungen zu deren realstiver Hinnahme stimulieren. Ein Angeklagter - um den Einwand auf unser Thema hin zu beleuchten -, der sich dem Gericht gegenüber lernwrillig, »kognitiv * verhielte, müßte ein Urteil wie ein unabwer.dbares Naturezeignis erfahren. Dann wäre es in kompakter, unmitrelbarer Fraglosigkeit »legitimierı «, abgenommen. - Die Wahl des theoretischen Bezugsrahmens ist eben nicht beliebig.

3. Dieser gegenaufklärerische Affekt zeigt sich noch klarer in Luhmanns Begriff der Legitimation, der niche zu trennen ist von dem der Institution. 39 Normen - so Luhmanns Ableitung des Institutions-Begriffs - verleihen Erwartungen zeitliche Stabilität, Dauer. Die Möglichkeit von Normenkonflikten macht weitere Leistungen erforderlich, dis der normativen Sollensqualitüt ihren noch subjektiven Charakter nehmen sollen. Zur zeitlichen Stabilisierung von Erwartungen (durch Normen in der subjektiven Perspektive) muß eine soziale Stabilisierung hinzutreten. Die Bedingung von sozialem Konsens, Integration ist die Institutionalisierung von Verhaltenserwartungen. Weil aber expliziter Konsens in einer komplexen Gesellschaft knapp ist, kann die Institutionalisierung von Verhaltenserwartungen jedoch nur noch bedeuten, daß Konsens als erwartbar unterstellt werden kann. »Die Funktion von Institutionen liegt in der Okonomie des Konsenses, und die Ersparnis wird hauptsächlich dadurda erreicht, daß der Konsens im Erwarten vorweggenommen wird, kraft Unterstellung fingiert und dann normalerweise gar nicht mehr honkret abgefragt werden muß. « ${ }^{30}$

Legitimität von Normen und Institituionen macht Luhmann deshalb nicht mehr an Prinzipien des guten Lebens fest. Historisch gesehen ist der Begriff der Legitimität »ins rein Faktische abgedrängt « (Verf., S. -7). Er bezeichnet nurmehr eine fakrische Uberzeugung. Während noch bei Max Weber der Legitimitätsbegriff aufgespalten wurde - entsprechend seiner empirischen und zugleich sinnverstehenden Soziologie - in eine Dimension von Legitimitätsprinzipien (etwa Staatszwedken, Werten, Postulaten, Prinzipien) und eine Dimension der empirischen Geltung, befaßt sich Luhmann nur noch mit der letzteren. Gleichwohl beansprucht auch er einen sinnverstehenden Ansatz. Der ${ }_{\text {Sinn }}$ e einer fakrisch konstatierten Uberzeugung erschloß sich Max Weber aus dem Bezug der subjektiven

9 Zum Begriff der Institutionalisierung s. zuletzr: N. Luhmann, Institutionalisierung - Funktion und Mechanismus im sozıalen System der Gesellschaft, in: Helmur Schelsky, $\mathrm{H}_{\text {o. }}$, Zur Theorie der Institution, Düsseldorf: Bertelsmann Unir.verlag, 1970, S. 27-is.

so Luhmann, Normen (N. 25), S. 4 r. 
Motivation (von dumpfer Gewöhnung bis zu zweckrationalen Erwägungen), in der Legitimität als seinsollend erlebt wird, auf Legitimitätsprinzipien. Den Handlungstypen, differenziert nach ihren jeweiligen Legitimitäts-, Glaubensvorstellungen, dem *Legitimitätseinverständnis $\alpha$, entsprechen deshalb verschiedene Herrschaftstypen mit jeweiligem Legitimitätsanspruch. Luhmann hingegen greift zum Verständnis des fakrischen Legitimitätsglaubens nich mehr auf Geltungsprinzipien zurück, scndern auf fakrische soziale Mechanismen, die eine »alternativlose Selbstverständlichkeit im Handeln sichern. Werte - traditionelle Legitimationsmittel - werden nicht etwa verleugnet, aber auf ihre soziale Funktion hin befragt. ${ }^{31}$ Es hönnte dann andere Mechanismen geben, wodurch dieselbe Funktion crfüllt werden kann. „Verfahren « soll ein solcher Mechanismus der Legitimation sein. Hieran müßte sich zeigen, ob Luhmanns Legitimations-Konzept lerzrlich als neue Ideologie der Fellachisierung - als Konsequenz der *Faktisierung « des Legitimitäts-Begriffs - fungiert und ob Legitimation tatsächlich ohne Bezug auf Werte, normative Begründungen konzipiert werden kann.

4. An der Gegenüberstellung von Legitimation sdurch Verfabren und „durch symbolische Implikation (S. 36) läßr sich die zweite Frage behandeln. Verfahren soll einen fakrischen Kommunikationsprozeß* bezeichnen, $*$ symbolische Implikation weise dagegen, wie juristische Begründungen etwa, eine snormative Sinnbeziehung a auf (S. 37, s. o. zit.) Vom logischen Status schließen sich also beide aus. Faktisch ergänzen sie sich: »Im Grunde muß jeder Legitimierungsvorgang, der sich nicht unmitrelbar auf die Uberzeugungskraft und den Morivwert der Rechtsnormen stützr, auf außerrechtlichen Mechanismen beruhen.* (S. 79) Die Frage ist dann einmal, welche Bedeutung juristischen Begründungen noch zukommen kann für die Lösung des Bezugsproblems: die Abnahme von Entscheidungen und die Absorption von aufkommendem. Protest; zum anderen: ob das Gerichtsverfahren in dieser seiner Funktion (die nicht die einzige ist) hinreichend ohne Bezug auf juristische Begründungsformen, ohne »normative Sinnbeziehung « überhaupt, begriffen werden kann.

a) Welche Bedeutung kann gerichtlichen Urteilsbegründungen zukommen? Richterliche Deduktionen müssen Plausibilität für sich in Anspruch nehmen können. Die logische Konsistenz ist dabei Elementarform überzeugender Begründungen. Ihre Plausibilierung * vollzieht sich aber nicht nur im Medium formaler Logik; juristische Dogmatik und Methodenlehren stellen darüberhinaus ein reichhaltiges Arsenal möglicher Begründungen, Topoi, Argumentationsinstanzen bereit. Diese Mittel sollen «überzeugen «: einmal die durch das Urteil unmittelbar Betroffenen. Hierbei zahlt sich gerade die scopiose Argumentation aus: es wird eine Vielzahl von Begründungen geliefert, deren Zusammenhang kaum mehr zu rekonstruieren ist, die vertrösten, obwohl sie verurteilen, die Distanzierungen gestatten, weil sie nicht die Persönlichkeit betreffen (wie etwa psychoanalytische Beurteilungen), sondern nur ein abstraktes Zurechnungssubjekt. ${ }^{32}$

31 Vgl. N. Luhmann, Grundrechte als Institurion, Berlin: Dunder \& Humblor, 1965. - Wenn z. B. Grundrechien eine Funktion, letzelich die der Aufrechterhaltung des gesellschaftlichen Differenzierungsgrades, zugesprochen wird, sie nicht mehr juristisch-hermeneutisch in ihrem Sinngehalt expliziert werden, bleibe an Normativem nur der strategische Hinwcis, daB, wer den bestehenden sozialen Differenzierungsgrad - wer auch immer den definiert (ohne dabei selbst wieder auf Grundrechte zurüdkgreifen zu können) - aufrechterhalten will, auch die entsprechenden Grundrechte anerkennen muß. Ahnlich lautet der letzte Grund für die Interpretation von Institutionalisierung: Komplexitäte der Gesellschaft. Das läßr sich nidhe allein, angesichts des historisch variablen Differenzierungsgrades\&, als anthropologisch notwendige Entlastung ausweisen.

s: Ein klassischer Mechanssmus der Protest-Absorption wurde in der gerichtlichen Behandlung von Streikenden und Gewerkschaften angewendet: einerseits wurde gegen $\$ 615$ BGB ein Kollek- 
Es gibt also verschiedene Medien, die zur Annahme mißliebiger Entscheidungen führen, die es jemandem ermöglichen sollen, sich mit einer Situation abzufinden, in der er ins Unrecht versetzt wurde. ${ }^{33}$

Neben der sprachlichen Uberzeugungsbildung bleibt dem Verurteilten die Möglichkeit der Berufung oder Revision, dic Möglichkeit, sich protestlos vom Urteil zu distanzieren, vielleicht der Trost cines knappen Stimmenverhältnisses. Die Befolgung enttäuschender Imperative erfolgt nicht rein verbal im plausiblen Fther; Rollenerwartungen, dem Juristen-Beruf gegenüber etwa, bestimmen ebenso die Motivlage der Rechtsunterworfenen: professionell imponierende, *imponierte « Argumente.

Richterliche Begründungen sind nicht nur an die Betroffenen adressiert. Sie sollen - und diese Funktion bildet sich historisch mit der juristischen Professionalisierung und Rationalisierung heraus - ebenso obere oder untere Gerichte überzeugen; sie sollen nachvollziehbar und überprüfbar sein. Deshalb ihre Darstellung in intersubjektiv-logischer Form. Also, nicht nur Verurteilte sollen Urteile abnehmen; das wäre zu eng, das macht Luhmann. Auch andere Instanzen sollen sie akzeptieren - und nicht nur gerichtliche; auch die akademische Doktrin nimmt Entscheidungen entgegen, überprüft sie (wie auch immer), versucht sie in die Rechtsentwidklung zu integrieren. Gerichtliche Begründungen liefern nicht nur der akademischen Doktrin verbindliche Argumentationsmuster; auch Exekutivorgane erhalten präskiptive Handlungsprämissen und Informationen über mögliche Rechtfertigungsstrategien. Darüberhinaus können sie in legislative Programme eingehen; sie konkretisieren und authentifizieren allgemeine moralische Vorstellungen beim Publikum; schließlich dürften sie der Entlastung der Richter selbst dienen: sie können ihre Motive glaubwürdig darstellen - zumindest für sich seibst.

Damit haben wir lediglich das breite Spektrum normativ-symóolischer Legitimationsweisen angedeutet; daraus hat sich Luhmann jedoch nur einen kleinen Teilbereich gewählt. Unsere Kritik kann sich also nicht nur darauf beziehen, was Luhmann unberüdksichtigt ließ; will sie immanent verfahren, so hat sie den Anspruch zu überprüfen, daß Legitimation durch Verfahren ohne »normativen Sinnbezug " in der soziologischen Theorie auskommen kann.

b) $\mathrm{DaB}$ auch andere Gerichtsinstanzen Adressaten von zu überprüfenden oder erneuten Vernandlungen zugrunde zu legenden Gerichtsentscheidungen sind, heißft für unsere Diskussion, daß Luhmann den »Abnehmerkreis« von zu legitimierenden Entscheidungen zu klein gewählt hat. Ohne normativen Sinnbezug könnte nicht erkannt werden, aus welchen Gründen die Gerichtshierarchie durchlaufen wird, welcher Kriterien sich Obergerichte bei der Beurteilung aufsteigender Fälle bedienen. Unser Einwand reicht weiter: mit steigenden Instanzen wird Luhmanns Verfahrenskonzeption unanwendbar. Sie behandelt vornehmlich mit interaktionistischen Termini ein "Situations-System «, ein »System* unter physischen Anwesenden.

Das unmittelbar dramatische Element geht in höheren Instanzen verloren. Die Schrifform ersetzt spontanes involvement«. Es wird zwar noch mitgespielt,

sivrecht anerkannt, im konkreten Fall aber wurden die Streikenden, weil sie ein solidarisches Kollektiv bildeten, zu Gesameschuldnern erklärt. (RGZ 107, 247 จ. 30. I1. 1923). Den Gewerkschaften wurde vom BAG ihre staatstragende Funkton atrestiert (das schmeichele), andererserts wurden im selben Urteil Streik und Aussperrung gleidhgestelle. (B.AGE I, 29I v. 28.1 .1955$)$.

is Erving Goriman hat einen ganzen Katalog zusammengestells: On Cooling the Mark Out: Some Aspects of Adaptation to Failure, in: Rose, ed., (N. I8), S. 4 s:-505. 
aber die Realität der Handlung schrumpft, wird zeitlich zerrissen, zerbröselt in juristischen Formeln. Es findet eine wachsende Verrechtlichung statt, nicht nur in der Terminologie. Zur Abnahme höchstrichterlicher Entscheidungen verhelfen dann ganz andere Mittel: juristisch wherrschende « Lehren, das Prestige hoher Richter, rüdkübersetzende Kunstfertigkeiten von Anwälten, einlullende Gemeinplätze (so viele Richter können sich doch nicht irren). Bei den sozial relevanten Entscheidungen also ist der Legitimations-Aspekt von "Verfahren * gering; der Primat kommt der inhaltlich-symbolischen Legitimation zu.

Aber selbst für den Aspekt, den Luhmann in den Mittelpunkt gerichtlicher Legitimation stellt, die Selbstbindung durch Rollenübernahme in einer Verfahrensgeschichte, würden wir daran festhalten, daß der legitimierende Effekt nicht ohne normativen Sinnbezug ausgemacht werden kann. Wir wenden uns zur Diskussion dieser These zunächst dem Status der Rollentheorie ${ }^{34} \mathrm{zu}$, mit deren Kategorien Luhmann die Konstitution einer Verfahrens-Realität beschreibt. Die Intersubjektivität von ego und alter bildet sich über die Identität von Symbolen (s. o.), insbesondere über die Komplementarität von Erwartungen und Normen. -Rollen « bezeichnen dann *Bündel von Verhaltenserwartungen", Rechte und Pflichten, mit denen sich Subjekte begegnen. Rollen sind nicht an Individuen gebunden, sondern an Positionen in einem Status-Geflecht, die bezogen und verlassen werden können. An Erwartungen sind, je nach ihrer Art, verschiedene Sanktionen, Verhaltenskontrollen geknüpft, über die definitorisch und exekutiv jeweilige Bezugsgruppen verfügen. Nach diesen Bezugsgruppen sind die Rollen selbst segmentiert. Der Begriff der Bezugsgruppe spiegelt die Erfahrung, daß wir uns in verschiedenen Umweiten unterschiedlichen Erwartungen konfrontiert sehen, unterschiedlich uns verhalten. Sein Sinn dürfte aber auch darin liegen, dem Begriff der normativen Erwartung einen empirischen Gehalt zu verleihen. Um eine hermeneutische Exegese von Rollenerwartungen - das sind ja keine manifesten Handlungen - zu vermeiden, ließen sich »relevante Bezugspersonen interviewen, deren Fragebogen-Meinung sich dann als "faktische " Erwartung ansehen ließe. ${ }^{35}$ Das zweite Mittel zur Transformation der rollentheoretischen Annahmen in empirische ergibe sich aus der Divergenz von manifestem Verhalten und normicrten Erwartungen (role performance / role obligation). Um die empirische Soziologie von der Interpretation geltenden Normen freizusetzen, wird eine Konformitätshypothese eingeführt: Menschen handeln rollenkonform. Normen tauchen dann nur noch in der blassen Bezeichnung »institutionalisiertes Wertesystem « auf. ${ }^{36}$ Aber das ist eine Operationalisierungs-Hypothese, die gleichsam Normen auf Handlungen als deren faktische Repräsentation projiziert, um den Erwartungs-Begriff darauf anwendbar zu machen; sie kann aber nicht die theoretische Differenz von Norm-Handlung verwischen.

34 Ich verweise nur auf die zwei klassischen deutschen Texte: Ralf Dahrendorf, Homo Sociologicus, Köln-Opladen: Westdeutscher Verlag $1969^{8}$ und Heinrich Popıtz, Der Begriff der sozialen Rolle als Element der soziologischen Theorie, Recht und Staat, Heft $331 / 332$, Tübingen: Mohr, 1967 (mit der relevanten Literatur).

ss Das Problem ist auch aus der juristuschen Diskussion um die Bestmmmung von Verhaltensstandards bekannt: ob Richter von sich aus bestummen, was etwa ein sehrbarer Kaufmanne sei oder ob eine andere Bezugsinstanz, vielleiche die Industrie- und Handelskammer, befrage werden soll. Die Schwierigketten liegen einmal darin, zu bestimmen, welche die relevante Bezugsgruppe ist, zum anderen bleibe das Problem der Interpretation erfrageer Meinungen bestehen.

30 Auch wenn der Roilenbegriff von vorneherein auf fakrisches Verhaiten begrenze wird - wie bei Popitz -, dringt hinterrüdks wieder das Problem ein, wie der Geltungsgrad - Geltung als faktische Befolgung - von Erwartungen definiert werden kann. (S. Popitz, N. 34, S. 24, :6, 42). Abweıchendes Verhalten kann dann nudit ohne Rüdgriff auf normatıve Kriterien, und wenn es auch nur Strafgesetze sind, eríaßi werden. 
Luhmarn lüßt die normativen Züge der Rollentheorie unexpliziert. Er bedient sidh ihrer nur in verdünnter Form zur Stützung der These, daß durch Rollenübernahme die Motivation des Handelnden eingefangen wird. Er betont die Nicht-Identität von Rolle und Aktor, nicht nur in der einen Richrung, daß ein Individuum sich in Rollen noch selbst als unverwechselbar darstellen kann, sondern auch, daß durch Rollenübernahme sich der Handelnde selbst festlegt, eine Verbindlichlieit eingeht, von der er nur um den Preis der Glaubwürdigkeir sich lossagen kann: doing is being. Das Rollenkonzept verwender Luhmann also lediglich zur Bezeichnung dieser formalen Identität, eines monologischen Verhältnisses des Aktors zu seiner Rolle, nicht aber zur Analyse der symbolischen Interation in Rollen Handelnder.

Aber ein solcher Formalismus kann nicht den Legitimations-Effekt von Verfahren erklären. Dieser wird eher dadurch erzielt, daß in einem komplementären, agonalen Prozeß eine eigene sinnhafte Realität, die Verfahrensgeschichte, aufgebaut wird, die die Beteiligten bindet. Diese Realität erfaßt Luhmann nicht mehr rein rollentheorecisch. Er greift hierfür vornchmlich auf Goffmans Analysen von Situationssystemen ${ }^{37}$ zurück. Deren Status ist ungemein schwer einzuschärzen. Goffman formuliert keine soziologischen Globalinterpretationen, er ziseliert kommunikative Komponenten von Situationen, denen eigene Realität zukommt, dadurch daß sie durch Relevanzen, Themen, Definitionen, Transformationsregeln dafür, wie die Eigenschaften der beteiligten Subjekte aufzufassen sind, von der weiteren Umgebung abgehoben sind. Bezeichnenderweise begann er seine Arbeiten mit dem Modell eines Insel-Lebens. Dem liegr I. die Annahme zugrunde, daß »Gesellschaft « niche durch ein institurionalisiertes Werte-System zusammengehaleen wird, daß sich vielmehr unsere Orientierungen situativ a erst ausprägen. Gofiman versieht uns mit Situations-Interpretationen, die unsere eingeschlifenen Verhaltensweisen transparent machen, uns zugleich, neben der empfohlenen Interaktionsbewußheit, Regeln an die Hand geben, wie wir unser Verhalten situations-angemessen winszenieren "können. Goffmans Arbeiten beinhalten also 2. eine kommunikative Meta-Ethik. Sie können als Vorschlag gedeutet werden, wie jemand, der dic nebulöse Unreflektiertheit mitmenschlichen Umgangs durchbrochen hat, gleichwohl, bei aller Bewußtheit kommunikativer Ambivalenzen und folgender Verunsicherung der Interaktion, noch Spontaneität, Engagiertheit, Ernsthaftigkeit darstellen kann. Es sind moralische Annahmen, die um den Begriff der Ich-Identität zentriert werden können. Sie betreffen das Verhältnis von Aktor und Rolle, die Selbstdarstellung als unvertretbare Individualität, das Paradoxon von reflektierter Spontaneitär. Eine Handlung ist solange unsere Identität davon betroffen wird - nicht bloße Erfüllung von Aufgaben, Zwedken. Unser Verhältnis zur Rolle und zu anderen Subjekten impliziert stets einen moralischen Aspekt. "Before a set of task-like activities can become an identy-providing role, these activities must be clothed in a moral performance of some kind. ${ }^{38}$ Die Verstrickung in Rollen und Geschichten ist ein

3: Goffmans wichtigste Arbeiten: The Presentation of Self in Everyday Life, New York: Doubleday, 1959 (dt. Ubersetzung: Wir alle spielen Theater, München: Piper, 1969); Asylums. Essays on the Social Situation of Mental Patuents and Other Inmates, New York: Doubleday, 1961 (auch als Penguin Book erschienen und danach zituert); Encounters. Two Studies in the Sociology of Interaction, Indianapolis-New York: Bobbs-Merrill, r96r; Stigma. Notes on the Management of Spoiled Identity, Englewood Clifis: Prentuce-Hall, 1963 (dt. Ubersetzung: Frankfurt: Suhrkamp, 1967); Bahavior in Public Places. Notes on the Social Organizzrion of Gatherings, New York: The Free Press, 1963; Interaction Ritual. Essays on Face-to-Face Behavior, Gurden City-New York: Doubleday (Anchor Books), r 967

ss Gofiman, Encounters (N. 37), S. Ior. 
moralischer Prozeß; sie trifft unsere Identität. Wir können Goffmans Arbeiten schließlich 3. als Sammlung von Klugheitsregeln für gekonntes Verhaiten interpretieren, als eine mit soziologischer Phantasie und Terminologie angereicherte Etiquette. Sie kann mit ihrer Betonung taktvollen Verhaltens, der Fähigkeit, Situationen aufrechtzuerhalten, das Gesicht zu wahren, ihre schichtenspezifische: bürgerliche Herkunft nicht verleugnen. ${ }^{39}$

Luhmann bedient sich also theoretischer Vorbilder, die wahrlich niche frei von normativen Implikaten sind. Die Betonung der Trennung von sfaktisch (im Sinne von wertfrei) und normative ist aber um so verwunderlicher, als Iuhmann selbst dieses Schisma für die Soziologie nicht anerlsennt, sich nicht einem Popper-Albert-Ideal empirisch-analytischer Wissenschaftlichkeit verschreibt. ${ }^{t 0}$ $\mathrm{DaB}$ er gleichwohl an entscheidender Stelle diese Trennung für sich einführr - um eine hermeneutische Exegese von Legitimationsprinzipien zu umgehen -, ist erklärlich allein dann, wenn wir seine ontologische Faktisierurg " des Legitimationskonzepts berücksichtigen. Die Interpretation von Legitimation als faktisches Geschehen - im Objektbereich - greit auf die Theorieebene über: Luhmann möchte Legitimation selbst noch wfaktisch", ohne "normativen Sinnbezug " betrachten.

Mit dem konsequenten Ausschluß von Legitimationsprinzipien zur Interpretation von Mechanismen für die Abnahme von Entscheidungen erweist sich Luhmann als Theoretiker der feinen juristischen Art, andere ins Unrecht zu versetzen, onne sie zu überzeugen.

c) Luhmann, der juristische Vokabeln zur Beschreibung juristischer Verfahren ausschließen möchte, läßt konsequent ein juristisches Mittel der Privatisierung von abweichendem, der Privatisierung von Protest gegen die Beurteilung abweichenden Verhaltens unberüdssichrigt. In *Legitimation durch Verfahren « wird die Konzeption individueller Schuld nicht erwähnt. An anderer Stelle (Normen, [N. 25] S. 45 f.) begriff er noch Schuld als eine *Enttäuschungserklärung, die es gestattet, das enttäuschende Ereignis auf sich selbst zu isolieren « (ebd. S. 46); allerdings bestand die Isolierung für ihn - in Abhebung von antiker Tragik darin, daß das enttäuschende Ereignis mnicht als unabsehbar weiterlaufendes Verhängnis« angeschen wcrde, das in Generationen fortwirke. Denn Schuld setze, als Maß der Strafe, den Folgen der Tat ein Ende. Die begrenzende Funktion von Scluld sicht Luhmann nur in ihrem Bezug zur Sühne. Damit kann sich eine Soziologie, die sich als Aufklärung versteht, nicht begnügen, nicht mit der theoretischen Verdoppelung der offiziellen Enträuschungserklärung, daß mit Ableistung der Strafe die Welt wieder heil sei. Sie könnte nicht die limitierende Wirkung von individueller Schuld im Hinblick auf soziale Verantwortlichkeit übersehen. Die Isolierung des enttäuschenden Ereignisses besteht ja nicht nur darin, daß der tragishe Faden nicht weiter gesponnen wird, sondern daß auch die Umwelt von Verantwortung entlastet wird: die Tat wird einem isolierten Subjekt mit bösen Motiven zugerechnet. Schließlich dürfte eine soziologische Analyse nicht in scheinbarer Wertfreiheit historisch variable Zurechnungsstrategien als mögliche Lösungen undiskutiert nebeneinander stellen: mythologischen Hexenglauben, die juristische Punktualisierung eines personalen Täters, wissenschaftliche, psydroanalytische Motivforschungen. Allen drei "Enträuschungserklärungen * liegen, und das sieht auch Luhmann (ebd. S. 45), normative Annahmen zugrunde. Aber von deren Explikation und Diskussion hält sich Luhmann 
zurüdk. Dabei würden nämlich die juristischen Zurechnungsformen rettungslos diskreditiert als Mittel der Ausblendung sozialer Verantwortlichkeit, der Kanalisierung gesellschaftlicher Aggressionen auf einen personalisierten Zurechnungspunkt.

d) Es geht in diesem vierten Abschnitt um den Nachweis, daß Luhmann seinen Anspruch, wVerfahren « und dessen Funktionen ohne normativen Sinnbezug zu deuten, nicht einlösen kann. Entweder bedient er sich Konzepten, die einen uneingestandenen normativen Sinn besitzen - es sei denn er spielt die Implikate durch Formalisierung herunter -, oder er spart Themen aus, die für die Funktion von Verfahren relevant sind, denen aber ein deutlicher Wertungscharakter eignet.

Als letzres Beispiel dafür diskuriere ich Luhmanns Modellvorstellung der Rechrsanwendung als eines in konditionaler Programmform repräsentierbaren Prozesses. Diese Programmform soll neben ihrer angeblichen Berechenbarkeit - ein Tatbestand löst, wenn er gegeben ist, eine feststehende Rechtsfolge aus - auch den Vorzug besitzen, den Anwendenden von den Folgen seiner Tätigkeit zu entlasten; er vollstreckt anonym, ohne persönliche Verstrickung und durch andere ersetzbar, eine allgemeine Norm. Meine These ist, daß Luhmann mit dieser Interpretation keine empirische Beschreibung des Rechtsanwendungs-Prozesses liefert, sondern das legitimierende Selbstverständnis der Juristen reproduziert. Das Subsumptions-Modell, auf das sich die konditionale Programmform zurückführen läßt, stellt eine inhaltliche Legitimation der Justizorganisation dar, nicht aber die Widergabe eines »faktischen Prozesses «. Es dient der Legitimation des Verfahrens.

Gerade Luhmanns hier ausführlich diskutierter Arbeit können wir zwei Thesen entnehmen, die der herkömmlichen juristischen Subsumptions-Vorstellung den Boden entziehen:

I. Rechtsanwendung ist ein kommunikativer Prozeß. Anwendung von Rechesnormen geschieht nicht monologisch, subsumptiv, sondern im intersubjektiven Austausch von Argumenten mit Plausibilitäts-Anspruch. ${ }^{41}$

2. Rechtsanwendung geschiebt innerbalb eines offenen Horizontes. Wenn es einmal zum Prozeß gekommen ist, erlauben Rechtsnormen kaum eine Voraussehbarkeir des Urteils; das Verfahren lebr ja von der Ungewißheit des Ausgangs, sonst würde es zum Ritual.42.

Luhmann hat zudem selbst die Verwendung des Modells einer programmierten Verbindung von Tatbestand und Rechtsfolge für die Interpretation der Herstellung richterlicher Entscheidungen verneint. "Der Schluß von einem Tatbestand auf eine Rechtsfolge ist für den Juristen die Endgestalt, in der er sein Arbeitsergebnis präsentiert, nicht aber ein Abbild oder Modell seiner faktischen Entscheidungstätigkeit. Die logische Form hat eine Darstellungsfunktion. ${ }^{43}$ In der Unterscheidung von Herstellung und Darstellung fällt die konditionale Programmform unter den Darstellungsaspekt. Ihm kommt die Funktion zu, Urteile übertragbar und nachprüfbar zu machen, sie durch den Neutralitäts-Mythos zu legitimieren. Die Interpretation der Herstellung von richterlichen Entscheidun-

11 Vgl. meinen Aufsatz, Klassenjustiz?, in: Kritische Justiz s/1969, S. 1-26.

4: Es ist bezeicinnend, daß diejenigen, die in liberaler Manier den Were von Rechesnormen auch in der Ermüglichung von Berechenbarke1t im institutionellen Rahmen sehen, zugieich Prozesse kritisieren. weil das Urteil schon zu Anfang feststehe. Rechtssicherheit heiße dann nur noch, daß im Verfahren alles smit rechten Dingen zugeht.

$43 \mathrm{~N}$. Luthmann, Recht und Auromation in der öffentlichen Verwaltung, Berlin: Dundker \& Humblor, I 966, S. sr. 
gen gelingt dagegen eher in einem thcoretischen Bezugsrahmen, wie ihn Luh$8 I$ mann gewählt hat zur Analyse von "Verfahren«: einem interaktionistischen; nur teilen wir nicht seine Ansicht, daß dieser frei von normativem Sinnbezug sei.

s. Wenn schon Luhmann durch die Trennung von Legitimation durch Verfahren und Legitimation durch symbolische Implikation einer Kritik des materiellen und formellen Rechts sowie der übrigen juristischen Begründungsmittel im Hinblick auf ihre legitimierende Bedeutung meint sich begeben zu können, so härte doch eine distanzierte Analyse der Bedingungen nahegelegen, die er für sein Legitimationskonzept in den Vordergrund stellt: die informellen Interaktionsnormen des Prozesses. Der letzte Punkt unserer Kritik betrift Luhmanns Legitimation von Verfahren durch Entproblematisierung der geriobtlichen Interaktion.

Jene informellen Interaktionsnormen gerinnen bei ihm zu unproblematischer Fakrizität, in die man sich nur verstridken kann. Der "sichere Verhaltensrahmen «, über den der Richter waltet, damit die Beteiligten sin Ruhe eine gute (eine nach den Maßstäben des Verfahrens gute!) Leistung vollbringen " (S. 86), dieser Verhaltensrahmen und seine Maßstäbe werden nicht auf ihre Legitimität befragt; es wäre eine inhaltliche Legitimität, die sich zu bewähren hätte an einem Modell herrschaftsfreier Kommunikation.

Freilich diskutiert Luhmann auch Elemente der kommunikativen Belastung und Asymmetrien, sowie deren mangelnde Kompensation im Gerichtsverfahren. Er betont die vor Gericht verschärfte Pflicht zur Konsistenz der Darstellung (S. 92); Außerungen sind bindend, werden ernst genommen und protokolliert. Die Entscheidungsempfänger und Zeugen werden als Informationsträger und Beweismittel behandelt, aber nicht als Personen anerisannt. Ihre Aussagen müssen unter Normen subsumierbar sein, sonst sind sie irrelevant. Die Entlastung von dieser Anspannung wäre möglich durch unpersönliches Handeln, das aber steht nur dem Richter zu; der nicht hauptberuflich Beteiligte ist zur Selbstdarstellung gezwungen, seine Persönlichkeit soll eingefangen werden. Sie könnte auch durch expressive Rollen-Distanz erreicht werden; aber das widerspräche der offiziellen Ernsthaftigkeit. Weitere Asymmetrien, neben denen der Entlastungsmöglichkeiten, bestehen in der Verteilung von EinfluB, auch darin, da $B$ das Gericht seine Meinung im Dunkeln beläßt, die anderen aber ihre öffentlich festlegen müssen. Für die einen sind Prozesse Routine, für die anderen ein außergewöhnliches Ereignis.

Luhmann begreift aber Verfahren nicht als systematisch verzerrte Interaktion. Statt die Asymmetrien an einem Modell unverstellter Kommunikation zu messen, verengt er seine Analyse auf das Problem der mehr oder weniger lernwilligen Abnahme von Entscheidungen. Selbst seine Vermutungen über entlastende Situationen sind noch geleitet von Kriterien erhöhter Lerneffizienz. Würde die strikte Programmierung wegfallen, abweichendes Verhalten nidit vorweg und frontal diskriminiert werden, könnten flexible Reaktionen erfolgen, Belohnungen für Lernerfolge nach individuellen Bedürfnissen verteilt werden, ließe sich die Überzeugungskraft der eigenen Argumente miterleben - dann könnten die Beteiligten auch besser »lernen «. Das macht das Ambivalente von Luhmanns Buch aus: daß es durchaus kritische Einsichten originell vermittelt, diese dann aber nicht in den knappen Bezugsrahmen einfügen kann. Der ist durch gegenaufklärerische Konzepte von Legitimation und Lernen für emanzipatorische Kritik blockiert. Ubrig bleibt in "Legitimation durch Verfahren * die Legitimation einer verfahrenen Situation. 
Das soziale Klima, in dem Luhmann sein Legitimationskonzept formuliert, scheint gekennzeichnet zu sein durch privatistische Entpolitisierung, weitgehende Außerfragestellung der gesellschaftlichen Institutionen. Sein Konzept einer modrigen Faktizität - ich sprach von Fellachisierung - hat also durchaus Realitätsbezug. Andererseits zeigt sich gerade bei der Justiz ein prekärer Mangel an Legitimationen, der spätestens seit den politischen Demonstrantenprozessen offenkundig wurde. Nur durch die Amnestie ist die Justiz weiterem Legitimationsdrudk entgangen. Luhmann bieter nun eine neue Legitimationsform an, eine, die ohne normative Legitimationsprobleme auszukommen hoff, sich nur auf die Reibungslosigkeir eines allein für Sozialtechnologen durchschaubaren Geschehens verlassen kann.

Das kritische Gegenmodell einer nicht gewaltsam verstellten Kommunikation setzte eine allgemeine Theorie der Kommunikation voraus, die auch systematisch pachologische Situationen erfaßt.44 Mein Versuch reicht nur so weir, daB ich einige bislang entwidkelte Kategorien auf die gerichtliche Interaktion anwende. Extrapolationen atf andere Komnunikationsbereiche werden vorsichtig angedeutet; das Modell wird aber nicht so weit universalisiert, daß Gesellschaft überhaupt idealiter als ungez wungene Sprechgemeinschaft erschiene.

Expressive Rollendistanz erwähnte Luhmann als ein mögliches Mittel der Entlastung von Konsistenzanforderungen in Verhalten und Darstellung, räumte ihr aber geringe Chancen angesichts der Notwendigkeit ernsthafter Verstridkung in die Verfahrensgeschichte ein. Nun lebten gerade die Prozesse gegen (studentische) Demonstranten von einer solchen Distanz den üblichen Rollenklischees gegenüber. In ihnen wurden die informellen Interaktionsregeln pioblematisiert - weniger die materiellen Gesetze; man berief sich ja aufs Grundgesetz. Hier stand die Legitimität von Verfahrensrollen zur Diskussion. w Wir empfanden uns da als Zuschauer, die gelegentlich eingriffen, wenn es uns $\mathrm{Spa} B$ machte. Und das war allerdings häufig... Mitspieler waren wir meist nicht, weil es nicht unser Spiel war, wir wären gar nicht auf den Gedanken gekommen, daß man solche Stïcke machen kann. Wir wurden es erst und dann mehr als Regisseure, als wir die Möglichkeiten erkannten, die uns geboten wurden. ${ }^{45}$ Statt sich zu verstricken, haben sich Angeklagte von dem Geschehen distanziert, das für sie als Szene, Marionettenspiel transparent wurde. Während die anderen an Rollenfäden hängend agierten, vermochte die Sensibilität der »abnormen Persönlichkeiten $\kappa$, die sie sein sollten, das $Z$ wanghafte jenes wsicheren Verhaltensrahmens« deutlich zu bezeichnen, das ein Tribunal für seinen reibungssollen Vollzug zur Bedingung hat.

Gerichtsverfahren als zerbrochene Kommunikation zu untersuchen, bieter sich besonders deshalb an, weil in ihnen Normen mit intersubjektivem Geltungsanspruch nach ihrer Verletzung wieder authentifiziert werden sollen.

4 Eine solche Theorie liegt allenfalls in Ansätzen vor. Sie beziehen sidh vor allem auf Familiensysteme, insbesondere soweit sie Sozialisationsstörungen bedingen. S.: Paul Watzlawıd, Janeth H. Bearin, Don D. Jadeson, Menschlide Kommunikation. Formen, Störungen, Paradoxien, Bern-Stuttgart: Huber, z 969 ; Gregory Bateson, Don D. Jadsson, Ronald D. Laing u. a., Schizophrenie und Familie, Frankfure a. M.: Suhrkamp, 1969; Hans Perer Dreitzel, Die gesellschaftichen Leiden und das Leiden an der Geselischaft. Vorstudien zu einer Pathologie des Rollenverhaltens, Sturtgart: Enke, r968; Jürgen Habermas, Thesen zur Theorie der Sozialisation, Manuskript der Vorlesung im Sommer-Semester $196 \$$ und die Arbeiten von Goffmann, insbesondere Asylums, S. auch: Dieter Claessens, Rolle und Machr, München: Juventa, $1970^{\circ}$; ders., Rollentheorie als bildungsbürgerlidhe Verschleierungsideologie, in: T. W. Adorno, Hg., Spätkapitalismus oder Industriegesellschaft?, Stuttgart: Enke, r 969, S. 270-279.

15 Rainer Langhans im SchluBwort zum Prozeb gegen ihn und Fritz Teufel, in: Klau mich, Volt.:ire Handbuch 2, Frankfurt 2. M. und Berlin 1968. 
Meine These lautet: die Intersubjektivität von Rechtsnormen wird in einem asymmetrischen, überhaupt einem pathologisdjen Prozeß konstituiert. Mit dem Begriff der Asymmetrie bezeichne ich die ungleiche Verteilung von Kommunikationschancen; der Begriff der Pathologie meint darüberhinaus - rollentheoretisch ausgedrückt - ein gestörtes Verhältnis von Norm, Rollenerwartung einerseits und Motiven, Bedürfnissen andererseits bei allen in einer Kommunikation befangenen. Einmal kann Normsḑwäche, Anomie vorliegen, so daß keine klaren motivischen Orientierungsmuster gegeben sind. ${ }^{46}$ - Relevant für Gerichtsverfahren dürfte eher der umgekehrte Fall sein: ein Normdruck, so rigide, daß institutionalisierte Erwartungen und subjektive Motive zu verschmelzen scheinen; die Situation wird engmaschig, die normativen Anforderungen total. - Die Rigidität von Normen ist abhängig von ihrer subjektiven Erfahrung. Der Rollendruck kann verstärkt werden durch Iob-Schwäche aufgrund von Sozialisationsmängeln. (Die Aussage ist nicht umkehrbar: ein starkes Ich hat nicht der, der alle Anforderungen verinnerlichen kann.)

Mit lediglich analytischem Anspruch unterscheide ich folgende Verzerrungen:

a) vorgängige Asymmetrie der Verfahrens-Situation (ungleiche Einflußchancen)

b) Asymmetrien im Verfahren (Siruationsherrschaft)

c) Pathologien der richterlichen Rolle (Beobachtungsposition: Normalität und Unverbindlichkeir)

d) Pathologie der Situation (Ernsthaftigkeit)

Ad a) Die vorgängige Ungleichheit im Strafverfahren ist bekannt. Die Rollen sind vorweg definiert, die Fronten abgestedk. Eine Seite hat das Thema bestimme ( $\gg$ Anklageschrift ), die andere wird dadurch frontal angegriffen, sie allein ist möglicher Zurechnungspunkt von Normabweichungen. Nicht das Auftauchen der Kraniche des Ibykus, das sind ja Zurechnungsvögel, und die gewissenhafte Selbstdefinition der Verbrecher macht die Szene zum Tribunal. Die Rolle des Abweichenden wird von Instanzen zugeschrieben, die zugleich-über-die Zwangsmittel zum Eintritt in die Situation und zu deren Aufrechterhaltung verfügen, für die ihre Tätigkeit Routine, nicht Schidksal ist. Der Angeklagte hat meist keine Zeit, die Situation kennenzulernen; zudem ist seine finanzielle und sprachliche Kompetenz meist so gering, daß seine Einflußmöglichkeiten auf ein günstiges Urteil vorweg beschnitten sind. Die Situation - ihre Themen, räumliche und zeitliche Gegebenheiten, Relevanzen, Sprachformen, Beteiligte - wird also einseirig inszeniert.

Ad b) Diese Asymmetrie wirkt sich in Verfahren selbst aus. Besonders das Gefälle in der Sprachkompetenz stellt, neben der unterschiedlichen Qualität anwaltschaftlicher Vertretung, ein objektiv diskriminierendes Element dar. In Unterschichten herrscht Unverständnis gegenüber der juristischen Sprachwelt, die sich den Schein des sensus communis gibt. Wenn sich ein Richter verständlich machen will, gibt er sich meist gnädig, freundlich-herablassend. Jovialität ist ein Zug von Statushöheren. Juristen ähneln Ärzten, die sich mit unverständlichen Fachausdrüdken am Krankenbett über die Gebrechen des Patienten unterhalten. Nach einem Terminus von Goffman vollziehen sie snon-person treatment 47? Und wem es vor dem juristischen Sprachfaszinosum die Stimme verschlägt, gilt als verstockt.

Die gerichtliche Situation soll nach Luhmann offen sein, damit sich die Beteiligten

\footnotetext{
46 Diesen Fall und seine möglichen Kompensationen behandele ich nicht; s. dazu Dreitzel (N. 44), S. $366 \mathrm{ff}$.
}

17 Goffman, Asylums (N. 37), S. 298. 
ungezwungen selber binden. Diese Offenheit - und das macht ihre Ambivalenz aus - ist abcr sters revidierbar durch richterliche Intervention. $Z_{\text {war }}$ soll der Richter Zurüdthaltung üben; seine Rolle erlaubt ihm aber jederzeit apodikrische Bestimmungen des Verlaufs. ${ }^{48}$ Er kann ohne vorherige Verständigung oder höfliche Andeutung Themen wählen, Relevanzen angeben - $\gg$ Ihr Privarleben interessierr mich nicht. « -, zuteilen, wer reden darf. Das bestimmt das Autoritäre der Richterfigur: daß bei aller Zurüdkhaltung die Möglichkeit zur Situationsbestimmung klar ist, daß er schweigt und sich doch ein Urteil bildet, während die anderen offen argumentieren, daß der Richter das letzte Wort haben wird. Schweigsame Interpretationsherrschaft ist Pascha-Manier. - Der Richter verfügt nicht nur über die Interpretationskategorien, er ist nicht nur Instanz, Bezugsperson, die normative Erwartungen definiert; zugleich kann er manifeste Sanktionsmittel handhaben. Richter besitzen in einem die Interpretationsherrschaft und die Herrschaft über Gewalt- und Diskriminierungsmittel ${ }^{49}$; und zwar im Hinblick auf allgemeine Rechtsnormen und Sanktionen, wie auch auf die informellen Interaktionsnormen des Verfahrens. Die werden gestützt durch die permanente Drohung mit Ordnungsstrafen, durch allgemeine Stilanforderungen (Kleidung, Anrede, Körperhaltung etc.). Die Freundlichkeit des Richters: man dürfe sich ruhig setzen, scheint noch autoritärer zu sein als das anonyme Gebot, vor Gericht zu stehen. Der Richter kann das Wort abschneiden, Gelegenheit geben, etwas zu sagen, psychiatrische Begutachtung anordnen, peinliche Fragen stellen, deren Adressat er nie zu sein braucht (»Fragen Sie nicht, das Gericht fragt! «)

Ad c) Richter sind die eigentlichen Objekte der traditionellen Rollentheorie. Der homo sociologicus ist im Grunde der homo juridicus. Für sie trifft die Konformitärsthese zu: Rechrsnormen, allgemeine soziale Erwartungen bilden zugleich die subjekriven, gesetzesgebundenen Morive. Richter sind taugliche Objekte einer Kritik der Rollentheorie. Sie legt nämlich ihren harmonistischen Annahmen ein pathologisches Randmodell zugrunde. Die strenge Anforderung an den Richter, »moralisches Subjekt « zu sein, für das persönliche Maximen zugleich allgemeine Gesetze sein können, zeigt pathologische Auswirkungen im aktuellen Rollenhandeln, also im Prozeß. Die interventionsbereite Regungslosigkeit des Richters verstehen wir nicht bloß als Erfüllung der Funktion eines neutralen Streitschlichters; eine dermaßen reduzierte Expressivität (zudem in emotionsgeladenen Situationen) setzt, um durchgehalten zu werden, bestimmte Charaktereigenschaften voraus. Nicht nur die Stellung im Prozeß legt dem Richter Zurückhaltung auf, als Akteur muß er bereits eine durch familiale und akademische Sozialisation aufgebaute Disposition mitbringen, die ihm die identitätsstiftende Ubernahme einer Beobachterrolle in Kommunikationen erleichtert.

Aus alltäglichen Kommunikationen kennen wir das ungute Gefühl, das entsteht, wenn wir Leuten begegnen, die eine Beobachterposition beziehen. Sie hören zu, tragen wenig zum Fortgang bei, sie gehen kaum eine Selbstdarstellung ein, legen sich nicht fest, lassen überraschend Bemerkungen fallen. Sie sind schwer einzuschätzen, verunsichern uns meist, mitunter erzeugen sie bei anderen sogar ein schlechtes Gewissen. Man meint, sich dauernd vor ihnen rechtfertigen zu müssen.

43 Ein Frankfurter Richter fuhr den Verteidiger an: Halten Sie den Mund! Setzen Sie sich hin! Der folgende Befangenheitsantrag wurde abgelehnt, weil es sich um sein temperamentvolles, aber adäquates Mittele handele. Nach: Frankfurter Rundschau v. 26. I. 1971.

40 S. dazu Drestzel (N. 44), S. 226, als Kennzcichen besonders sdichter, Situationen.

50 Das geschieht natürlich alles unter der offziellen Legitumation der Wahrheitsfindung, der gerechten Behandlung der Betroffenen. Goffman hat für psychiatrische Instirutionen die Deformicrungen unter der Dedke des therapeutischen $\gg$ Anstaltszwedssa aufgezeigt. S. Asylums (N. 37$),$ S. $281 \mathrm{ff}$. 
- Der Richter hat diese Einstellung professionalisiert. Er kann sich jeder Kommunikation, Rückfragen z. B. entziehen. Ich vermute, daß seine Beobachterrolle vornehmlich zwei judizielle Qualitäten stützt: einmal die Fühigkeit, andere ins Unrecht zu versetzcn, ohne sie kommunikativ zu überzeugen. Wer Handlungen anderer vorgängig als mögliche Abweichungen einschätzen kann, wer sich zudem selbst als Hüter der Normalität begreift und damit die Erwartungen anderer ihm gegenüber derart hoch ansetzt, daß sie gar nicht zu erfüllen sind, für den sind Recht und Unrecht klar verteilt. Beim Richter bleibt diese Perspektive niche privat, er kann ihr durch seine Verfügung über Interpretations- und Gewaltmittel Verbindlichkeit verschaffen. - Daß überlegenes Schweigen als adäquate Darstellungsform von sich als einem "normalen « Wesen eingeschätzt wird, setzt voraus, daß expressive Selbstdarstellung als prekäres Wagnis eriebt wird. Die Angst sich Blößen zu geben, angesichts überstarker Normalitätsanforderungen an sich selbst, wird stillgestellt in neurotischer Regungslosigkeit, und beruflich anerkannt. Der Mangel an flexiblem Umgang mit Gewissensforderungen, selten gelernt und spätestens im juristischen Studium ausgetrieben, führt zu Kristallisierungen im Verhalten. Erträglich wird diese Erstarrung, weil sie als richterliche Tugend gepriesen wird, weil sie, in Verbindung mit der Situationsherrschaft, narziß tische und auch sadistische Befriedigung gestatter.

Neben dieser Fähigkeit, sich durch beobachtendes und organisatorisch abgesichertes Schweigen als normal darzustellen, andere durch Ǔberprägnanz der Normen mit Skrupeln zu belasten, fördert die Beobadıterposition des Richters auch seine hermeneutische Fertigkeit, Außerungen anderer zu verstehen, obne sie als verbindlich $z u$ bebandeln. Unter dem Schein des $»$ Sichhineinversetzens* durch aufmerksames Zuhören gerinnen die Beiträge der Beteiligten zu bloßen Beweismitteln, deren gerichtliche Einschätzung dunkel bleibt. Ohne Vergewisserung durch reziproke Verständigung übers Gesagte zappelt man sich ab. Metakommunikation, die Kommunikation übers Kommunizierte, wird unmöglich. Beobachter geben sich verständnisvoll, sind aber unverbindlich.

Das totale Aufgehen in einer Rolle (»embracement«) zeitigt zahlreiche Verhaltensschwierigkeiten für den Richter. Wer sich als Vertreter der Normalität und zugleich unverbindlich anderen gegenüber versteht, dem fällt es schwer, die Balance von Distanz und Nähe im Umgang mit anderen zu wahren. Wer die Ideale von Gerechtigkeit und Wahrheit gesichert hinter sich weiß und sich gar nicht in die Rolle der anderen zu versetzen braucht, der vermag Grenzen des Peinlichen kaum zu erkennen; der kann sein Interesse an der Privatheit des anderen verleugnen und zugleich delikate Fragen über dessen Liebesleben stellen (z. B. in der Kommune I); der kann mit größtem Gleichmut und höflich vier Jahre Ordnungsstrafe *ausspredien «51. Die Oberflächlichkeit der Motivforschung mischt sich allzu oft mit lüsterner Neugier. Rücksicht auf die Selbstdarstellung des Angeklagten: Takt ist kaum möglich; das Verfahren ist eher auf dessen Rollen-Tod aus ${ }^{52}$. Nicht in verletzlicher Intersubjektivität wird versucht, eine Geschichte zu

31 Im Prozeß gegen Bobby Seale wurde klar, wie sid der höflicher Ausdrudksweise bedienen kann, der den Gewaltapparat hinter sich hat. Das Nichteingehen des Richters auf Bobby Seales Wunsch, sich selbst zu verteidigen, sein Interesse an der raschen Fortführung der Verfahrensgeschidhte, angesichts dessen die Insistenz der Verteidigung als bloße -Verzögerungstaktik erscheint - das zeigt den kommunikativen Extrempunkt einer zerbrochenen Interaktion. Die der Kommunikationsstruktur immanente Gewalt manifestierte sich in der höflichen Anordnung, zunächst Bobby Seale zu iesseln und zu knebeln, dann vorerse für vier Jahre ins Gefängnis zu stedken. (S. dazu: Der ProzeB gegen Bobby Seale. Rassismus und politische Justız in den USA, hg. v. Bladk Panther Solidaritärskomitee, Frankfurt 2. M. (1970), Adalbertstr. 6.)

32 S. Goffman, N. 33, S. 503: die barbarische Zeremonie des Strafprozesses ist so angelege, daB der Verurteilte sein Gesicht niche wahren kann. 
rekonstruieren. Der Richter bleibr im Grunde ganz bei sich, $1 \ddot{3} \mathrm{\imath}_{\mathrm{t}}$ sich nicht in den intersubjektiven Aufbau einer Verständigung ein. Wenn er sich ironisch von seiner Rolle distanzieren möchte, macht er sich meist lächerlich.

Dieses beobachtend-monologische Verhalten des Richters dürfte die sinnvolle Basis für die Deduktions-Vorstellungen in juristischen Methodenlehren sein. Rechtsanwendung kann als einsamer Prozeß nur interpretiert werden, weil der Richter sich der Kommunikation, die ein Verfahren ist, entieht. Logische Subsumption ist hier nicht von Herrschaft zu trennen.

Wessen Beruf es ist, harmonische Weltvorstellungen durchzusetzen, dem muß jede Abwcichung als drohendes Chaos widerfahren. Die Welt erscheint dem Juristen als beängstigendes Potential möglicher Regelverstöße. Der Umgang mit einer *bösen Welt« dürfte ein Grund für das bei Juristen häufig anzutreffende Wehleid sein ${ }^{53}$, das nicht mehr durch die Hoffnung auf eine heile Transzendenz gemildert ist.

Beklagt werden auch Probleme der Rollentrennung, also Schwierigkeiten, dic sich mit dem Eintritt in neue Situationen ergeben. Wenn der Richter seine Robe ausziehen, den erhöhten Sitz räumen muß, Privatmensch wird, der all seiner beruflichen Verhaltenssicherungen entledigt ist, wenn er das Wagnis der Selbstdarstellung eingehen, sich die Einschätzung anderer erst erarbeiten muß (nur im Karneval könnte er ungefährdet mit Talar »im Freien « herumlaufen) - dann werden Kommunikationen für einen eingefleischten Beobachter delikat. Oder er spielt den Richter im Alltag weiter, in $n$ neuen Kleidern ${ }^{35}$

Apropos Rollentrennung: ich schlage vor, das Verhalten von Fritz Teufel, Langhans u. a. als das von anständigen Bürgern zu interpretieren, die nur nicht aus der Alltagsrolle in die Verfahrensrolle des Angeklagten schlüpfen können. Als anständige Bürger sind sie es nicht gewohnt, von jemandem, der sich ihnen nicht einmal vorgestellt hat, biographisch befragt zu werden. Sie empfinden die Aufforderung, gefälligst aufzustehen, als Unverschämtheit. $\mathrm{Daß}$ man den anderen nicht unterbricht, haben sie in der Kinderstube gelernt. Wer würde nicht ungebalten über die Rüpeleien der Richter - wenn sie nicht Richter wären.

Ad d) Die rigiden Normanforderungen, denen der Richter unterworfen ist, erstrecken sich auf die übrigen Verfahrensteilnehmer. Uber die Situation breitet sich eine Atmosphäre starrer Ernsthaftigkeit, die kaum eine Distanzierung erlaubt: weder eine defensive Distanzierung (man möchte nicht auf seine Rolle fixiert werden), noch eine Distanzierung von einer überlegenen Position aus, um die Untergebenen nicht zu stark zu verunsichern. Das Gericht paßt auf; von ihm beobachtet muß man sich konzentrieren, vor Mißverständnissen hüten. ${ }^{35}$ Man

53 Vgl. Walter $O$. Weyrauch, Zum Gesellschaftsbild des Juristen, Neuwied-Berlin: Luchterhand, 1970 , S. 295 ff.

34. Der Herr Schwerdtner z. B., der hier doch ganz eindrudksooll aussieht -, als ich ihn das erste Mal auf dem Flur draußen sah, war er nur noch ein kleiner Mann mit großen Ohren und er war sehr schredkhaft. Man kommt sich getüuscht vor.e (Langhans, N. 45 ).

33 Nach Luhmann (N. 25, S. ${ }^{6}$ ) besteht die richterliche Funktion darin, durch absichtsroll subtiles Mißverstehen der Parteien normativen Streit routinemäßig in Dissens zu transformieren, in eine lediglich kognitive Divergenz zu verwandeln. Zumindest müssen die Beteiligten sich vor Geridt im kognitiven Stil verhalten, müssen sich vernünftig und lernbereit (wenngleich unüberzeugt) geben, müssen sich wechselseitig in Rollen anerkennen und so tun, als ob es nur um sichtige -Erkennenise von Tatsachen oder Rechtsnormen gehe. Ihr aufgestörtes Rechesgefühl komme nicht oder nur als Störung des Yerfahrens zu Wort. Er beruft sich dafür auf Vilhelm Aubert, Competition and Dissensus: Two Types of ConAict and of Conflict Resolution, in: The Hidden Society, Totowa: The Bedminster Press, 1965, S. 98 f. Von einer bloß kognitiven Divergenz ist bei Aubert aber keine Rede. Der rechtliche Konflikt von Interessen kann sich leicht zu einem Dissens über Werte wandeln. Die Unterdrükung expressiver Momente läßt den Schein von oLernbereitschafte (wie bei braven Schülern) aufkommen. 
kann sich nicht durch Trivialitäten, Blödeleien, Metaphorik entlasten; man wird dingfest gemacht, wörtlich genommen. Das reichhaltige Spcktrum möglicher Kommunikationsmodi wird auf sprachlichen Ausdruck, Verbalismus ${ }^{58}$ reduziert. Manifester Rückzug ist jederzeit dem Gericht möglich: es kann sich ins Beratungszimmer flüchten, wenn die Situation zu belastend wird, kann sich auf seiner Hinterbühne ("badk stage": Goffman) wieder in Szene setzen. Wer in einer Situation bleiben muß, die aufrechtzuerhalten er nicht bereit ist, kann trotzig werden, rebellisch, komisch. Abcr diese symbolischen Distanzierungen werden unterbunden, wahrscheinlich nicht, wcil sie selbst das Verfahren stören, sondern weil sie die Aufforderung enthalten, daß der andere auch aus seincr Rolle treten soll und erst die richterliche Verstörtheit angesichts einer solchen »Zumutung « löst die "Störung « aus.

Daß Luhman den Rollenbegriff eigentümlich formal verwendet, symbolische Bezüge etwa ausschaltet, dürfte sidh damit erklären lassen, daß nicht-professionell Beteiligte in der Tat meist nicht verstehen, was sich vor ihren Augen abspielt. Ihre "Rollenübernahme" baut keine Identität für sie auf (es gilt nicit: doing is being); es wird über sie hinweg verhandelt und subsumiert. Nur der gewaltsam gestützte Schein des Funkisionierens und das sprachlose Unverständnis der Verfahrensmystik binden.

Rollendistanz wird restringiert; in ernsthafter Arbeit sollen sich die Beteiligten weiter verstricken. Meta-Kommunikation ist kaum möglich; die Verständigung über die wechselseitige Einschätzung von Beiträgen, des bisherigen Verlaufs ist meist Indiz für einen sich anbahnenden Vergleich, das konsentierte Ende der Geschichte.

Richter können Kollegen im Alltag begegnen, die nicht ihren Beruf teilen; aber ihre Kommunikationsweise. Wir hatten sie bereits oben skizziert: sie halten sich aus Diskussionen heraus, wollen sich nicht festlegen, versuchen die Last der Selbstdarstellung anderen zu überantworten, konfrontieren dabei andere mit hohen Standards von Plausibilität, Exaktheir. Sie vermeiden laxe Formulierungen, fürchten mit den kleinsten Fehlern ihr Gesicht zu verlieren - Symptome mächtiger neurotischer Anforderungen an einen selbst. Die Dogmatik des Alltagslebens kennt, wie die Juristen, Zurechnungstridks: daß der und der die Schuld habe, sind typische Beispiele für eine Problembehandlung, die die eigenen Erwartungen, weil sie normal seien, stabil durchiält und den verletztenden Tatbestand beim anderen festmacht. Damit wird im Vorgriff über Recht und Unrecht entschieden. Mit der Fixierung yon Tatbeständen bei anderen und deren Verknüpfung mit Rechtsfolgen begibt man sich jeder Selbstproblematisierung und ist nur noch zu Symptomkuren fähig. Der Richter, angeblich konditional programmiert, ist nur der institutionalisierte Fall eines allgemeinen Handlungsmusters.

Das Modell gerichtlicher Interaktion läßt sich auf Interaktionen überhaupt erweitern. Der Fall einer in antagonistischen Interessen und Strategien zerbrochenen und durch pathologische Rollen gewaltsam aufrechterhaltenen Interaktion kann als Gegenbild einer idealisierten gelungenen Kommunikation gelten. Das wäre, ohne daß wir bereirs anuähernd über taugliche Kategorien dafür verfügten, eine Sinuation, in der die pragmatischen Bedingungen vernünftigen Redens erfüllt wären. Ich-Identität könnte sich in Meta-Kommunikation, RollenDistanz, der flexiblen Reziprczität von Erwartungen und Bedürfnissen entfalten. Freilich, sie wäre gezeichner rom Mangel jeder moralischen Perspektive: die

ss Vgl. Bateson u. a. (N. 44), S. 20. 
Probleme von Gewalt und Ideologie, die "sittlicha institutionellen Geltungsbedingungen liegen jenseits dessen, was direkt durch den "guten Willen «, den kommunikativ bewußten Vorsatz erfaßt werden kann. Deshalb wären meine Ausführungen als Plädoyer für ein »Rechrsgespräch « nur mißzuverstehen. Solange die objektive Funktion der Justiz (die durch Interaktionsanalysen nicht erschlossen werden kann) darin besteht, Normen ungebrochen durchzusetzen, kann dies nur durch die reale Subsumption der Motive der Abweichenden und der Abweichenden selbst geschehen. Wer straft, kann nur zynisch die Asymmetrie auf Zeit verschleiern. Freisler hatte die strafenden Konsequenzen seines Handelns offen in seinem Kommunikationsstil eingcholt. Die kritischen Rufe, Richtern - gar in gruppendynamischen Seminaren - psychologische Sensitivität zu vermitteln - als hätten sie vergessen, wie man sich schlicht im Alltag verhält können nur verstanden werden als Versuch, die Kommunikationsherrschaft von Richtern stilvoll zu perfektionieren. Sie könnten, mit Charme, Herrn Müller, der einst als Angeklagter angesprochen wurde, bitten, sich doch die Handschellen vom $\mathbb{W}$ achtmeister wieder anlegen zu lassen.

Gegen Luhmanns Verengung der Perspektive auf Verfahrensmechanismen, die zur Abnahme von Entscheidungen führen, habe ich versucht, den Blids auf die inhaltliche Legitimität dieser Mechanismen zu lenken. "Verfahren " galt uns eher als pathologische Interaktion. Damit ergeben sich Uminterpretationen zentraler Begriffe Luhmanns: die "Offenheit" der Situation ist fundiert in der unverbindlichen Beobachterrolle des Richters; die Lernbereitschaft der Agierenden ist eher ein durch die stidkige Ernsthaftigkeit erzeugter Schein; die »faktische« Rollenverstrickung gelingt durch sprachlose Ohnmacht gegenüber dem juristischen Sprachzauber.

37 Nach Fertigstellung des Artikels erschien eine Kritik Josef Essers am hier diskutierten Werk Luhmanns. (J. Esser, Vorverständnis und Methodenwahl in der Rechtsfindung, Frankfurt a. M.: Athenäum, 1970, S. 202-213) - Esser kritisiert an Luhmanns Lernkonzept, daB in ihm abstrahiert wird von einsichtsvoller Oberzeugungsbildung und kommunikativer Internalisierung - bei Betroffenen und Richtern. Auch er fragt, ob die symbolische Arbeit des Juristen nur das, Lerneni erleichtern soll. Auf dem Hintergrund von Essers Thema: Methode und Ergebniskontrolle in der richerlichen Praxis, wird Luhmanns Eliminierung inhalelicher Wahrheirskriterien und Legirimierungsfakroren noch deurlicher: Luhmann abstrahiert, indem er die hermeneurische Problematik übergehr, vom Interessenverständnis des Rechtsanwendenden (S. 206). Nur deshalb kann Luhmann für eine subsumptive Anwendung plädieren. Esser zeige zudem am Rationalitärskonzept in Luhmanns Analysen deren Charakter als instrument interessenblinder Institutionsdarstellung * (S. 209).

Allerdings kann ich nuche Essers Annahme teilen, daß Luhmann vom Modell des Zivilprozesses, zudem von einer veraltecen Vorstellung, ausgehe und auch niche sein günstiges Richterbild, das ihn rorschnell die Rechtsfindung als Kommunikationsprozeß rekonstruieren läßr. Indem er Interessen und Ideologien in den Köpfen der Richter, ihrem , Vorverständnise ansiedelt und nicht mehr auf sozio-ökonomische Prozesse zurüdkbezieht, kann er auf $\$$ Kommunikationsbereitschaft \& und Lernprozesse durch Konflike und Konfliktverständnis bei Richtern hoffen, die ein Akzeptieren gemeinsamer Maßstäbe erlauben sollen. Dann würde $\gg$ Recht als wirklich autonomer Faktor a auftreten, dem es um's *Vertrauen [als hätte Luhmann nicht selbst in einer Arbe:t über -Vertrauenc diesen Begriff als kritischen endgültig diskreditiert; H. R.I der ,Betroffenenc in einer allseitig ernst genommenen Bindung an konsentierte Rechtlichkeitskriterien und deren effiziente justizförmige Kontrolle im Konfliktfalle gehe (S. 21 I). Der hermeneutische Optimismus, den Esser gegen Luhmanns technokratische Optimierungen ins Spiel bringr, unterliegr dem Mißverstïndnis, als ob die sozialen Interessen, Ideologien und Gewaltverhältnısse, nachdem sie nur noch in svorverstïndigrene Köpfen ausgemacht werden, durch Explikation des Vorverständnisses in einer durch Kommunikationsbereitschaft und Ehrlidkeit gestützten DialogSituation sid auflösten in zuangloser Interaktion. Hicr treten Psychoanalyse und KlassenTheorie in ins Rectie. 